\title{
The Mathematical Foundations of General Relativity Revisited
}

\author{
Jean-Francois Pommaret \\ CERMICS, Ecole des Ponts ParisTech, Marne-la-Vall_ee Cedex 02, France \\ Email: jean-francois.pommaret@wanadoo.fr, pommaret@cermics.enpc.fr
}

Received May 13, 2013; revised June 19, 2013; accepted July 29, 2013

Copyright (C) 2013 Jean-Francois Pommaret. This is an open access article distributed under the Creative Commons Attribution License, which permits unrestricted use, distribution, and reproduction in any medium, provided the original work is properly cited.

\begin{abstract}
The purpose of this paper is to present for the first time an elementary summary of a few recent results obtained through the application of the formal theory of partial differential equations and Lie pseudogroups in order to revisit the mathematical foundations of general relativity. Other engineering examples (control theory, elasticity theory, electromagnetism) will also be considered in order to illustrate the three fundamental results that we shall provide successively. 1) VESSIOT VERSUS CARTAN: The quadratic terms appearing in the "Riemann tensor" according to the "Vessiot structure equations" must not be identified with the quadratic terms appearing in the well known "Cartan structure equations" for Lie groups. In particular, "curvature + torsion" (Cartan) must not be considered as a generalization of "curvature alone" (Vessiot). 2) JANET VERSUS SPENCER: The "Ricci tensor" only depends on the nonlinear transformations (called "elations" by Cartan in 1922) that describe the "difference" existing between the Weyl group (10 parameters of the Poincaré subgroup +1 dilatation) and the conformal group of space-time ( 15 parameters). It can be defined without using the indices leading to the standard contraction or trace of the Riemann tensor. Meanwhile, we shall obtain the number of components of the Riemann and Weyl tensors without any combinatoric argument on the exchange of indices. Accordingly and contrary to the "Janet sequence", the "Spencer sequence" for the conformal Killing system and its formal adjoint fully describe the Cosserat equations, Maxwell equations and Weyl equations but General Relativity is not coherent with this result. 3) ALGEBRA VERSUS GEOMETRY: Using the powerful methods of "Algebraic Analysis", that is a mixture of homological agebra and differential geometry, we shall prove that, contrary to other equations of physics (Cauchy equations, Cosserat equations, Maxwell equations), the Einstein equations cannot be "parametrized", that is the generic solution cannot be expressed by means of the derivatives of a certain number of arbitrary potential-like functions, solving therefore negatively a 1000 \$ challenge proposed by J. Wheeler in 1970 . Accordingly, the mathematical foundations of electromagnetism and gravitation must be revisited within this formal framework, though striking it may look like. We insist on the fact that the arguments presented are of a purely mathematical nature and are thus unavoidable.
\end{abstract}

Keywords: General Relativity; Riemann Tensor; Weyl Tensor; Ricci Tensor; Einstein Equations; Lie Groups; Lie Pseudogroups; Differential Sequence; Spencer Operator; Janet Sequence; Spencer Sequence; Differential Module; Homological Algebra; Extension Modules; Split Exact Sequence

\section{Introduction}

The purpose of this paper is to present an elementary summary of a few recent results obtained through the application of the formal theory of systems of ordinary differential (OD) or partial differential (PD) equations and Lie pseudogroups in order to revisit the mathematical foundations of general relativity (GR). More elementary engineering examples (elasticity theory, electromagnetism (EM)) will also be considered in order to illustrate the quoted three fundamental results that we shall provide. The paper, based on the material of two lectures given at the department of mathematics of the university of Montpellier 2, France, in may 2013 and Firenze, Italy, in june 2013, is divided into three parts corresponding to the different formal methods used.

PART 1: In 1880 S. Lie (1842-1899) studied the groups of transformations depending on a finite number of parameters and now called Lie groups of transformations. Ten years later he discovered that these groups are only examples of groups of transformations solutions of linear or nonlinear systems of ordinary differential (OD) 
or partial differential (PD) equations which may even be of high order and are now called Lie pseudogroups of transformations. During the next fifty years the latter groups have only been studied by two frenchmen, namely Elie Cartan (1869-1951) who is quite famous today, and Ernest Vessiot (1865-1952) who is almost ignored today. We have proved in many books and papers that the Cartan structure equations have nothing to do with the Vessiot structure equations still not known today. Accordingly, the quadratic terms appearing in the Riemann tensor must not be identified with the quadratic terms appearing in the well known Maurer-Cartan equations for Lie groups. In particular, curvature + torsion (Cartan) must not be considered as a generalization of curvature alone (Vessiot).

PART 2: Though we consider that the first formal work on systems of PD equations is dating back to Maurice Janet (1888-1983) who introduced as early as in 1920 a differential sequence now called Janet sequence, it is only around 1970 that Donald Spencer (1912-2001) developped, in a quite independent way, the formal theory of systems of PD equations in order to study Lie pseudogroups, exactly like E. Cartan did with exterior systems. However, all the physicists who tried to understand the only book "Lie equations" that he published in 1972 with A. Kumpera, have been stopped by the fact that the examples of the Introduction (Janet sequence) have nothing to do with the core of the book (Spencer sequence). We may say that the work of Cartan is superseded by the use of the canonical Spencer sequence while the work of Vessiot is superseded by the use of the canonical Janet sequence but the link between these two sequences and thus these two works is not known today. Accordingly, the Spencer sequence for the conformal Killing system and its formal adjoint fully describe the Cosserat equations, Maxwell equations and Weyl equations but general relativity (GR) is not coherent with this result because we shall prove that the Ricci tensor only depends on the nonlinear transformations (called elations by Cartan in 1922) that describe the "difference" existing between the Weyl group (10 parameters of the Poincaré subgroup +1 dilatation) and the conformal group of space-time (15 parameters).

PART 3: At the same time, mixing differential geometry and homological algebra but always supposing that the reader knows a lot about the work of Spencer, V.P. Palamodov (constant coefficients) and M. Kashiwara (variable coefficients) developped "algebraic analysis" in order to study the formal properties of finitely generated differential modules that do not depend on their presentation or even on a corresponding differential resolution, namely the algebraic analogue of a differential sequence. Using double duality theory, we prove that, contrary to other equations of physics (Cauchy equations,
Cosserat equations, Maxwell equations), the Einstein equations cannot be "parametrized", that is the generic solution cannot be expressed by means of the derivatives of a certain number of arbitrary potential-like functions, solving therefore negatively a $1000 \$$ challenge proposed by J. Wheeler in 1970.

The new methods involve tools from differential geometry (jet theory, Spencer operator, $\delta$-cohomology) and homological algebra (diagram chasing, snake theorem, extension modules, double duality). The reader may just have a look to the book [1] (review in Zbl 1079. 93001) in order to understand the amount of mathematics needed from many domains.

The following diagram summarizes at the same time the historical background and the difficulties presented in the introduction:

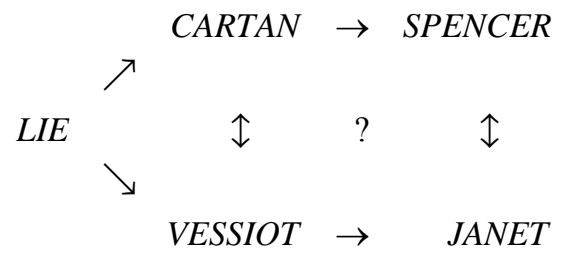

Roughly, Cartan and followers have not been able to "quotient down to the base manifold" [2,3], a result only obtained by Spencer in 1970 through the nonlinear Spencer sequence [4-7] but in a way quite different from the one followed by Vessiot in 1903 for the same purpose $[8,9]$. Accordingly, the mathematical foundations of mathematical physics must be revisited within this formal framework, though striking it may look like for certain apparently well established theories such as EM (J. C. Maxwell, 1864) and GR (A. Einstein, 1915).

\section{First Part: From Lie Groups to Lie Pseudogroups}

If $X$ is a manifold with local coordinates $\left(x^{i}\right)$ for $i=1, \cdots, n=\operatorname{dim}(X)$, let $\mathcal{E}$ be a fibered manifold over $X$, that is a manifold with local coordinates $\left(x^{i}, y^{k}\right)$ for $i=1, \cdots, n$ and $k=1, \cdots, m$ simply denoted by $(x, y)$, projection

$$
\pi: \mathcal{E} \rightarrow X:(x, y) \rightarrow(x)
$$

and changes of local coordinates

$$
\bar{x}=\varphi(x), \bar{y}=\psi(x, y) .
$$

If $\mathcal{E}$ and $\mathcal{F}$ are two fibered manifolds over $X$ with respective local coordinates $(x, y)$ and $(x, z)$, we denote by $\mathcal{E} \times_{X} \mathcal{F}$ the fibered product of $\mathcal{E}$ and $\mathcal{F}$ over $X$ as the new fibered manifold over $X$ with local coordinates $(x, y, z)$. We denote by

$$
f: X \rightarrow \mathcal{E}:(x) \rightarrow(x, y=f(x))
$$


a global section of $\mathcal{E}$, that is a map such that $\pi \circ f=i d_{X}$ but local sections over an open set $U \subset X$ may also be considered when needed. We shall use for simplicity the same notation for a fibered manifold and its set of sections while setting $\operatorname{dim}_{X}(\mathcal{E})=m$. Under a change of coordinates, a section transforms like

$$
\bar{f}(\varphi(x))=\psi(x, f(x))
$$

and the derivatives transform like:

$$
\begin{aligned}
& \frac{\partial \bar{f}^{l}}{\partial \bar{x}^{r}}(\varphi(x)) \partial_{i} \varphi^{r}(x) \\
& =\frac{\partial \psi^{l}}{\partial x^{i}}(x, f(x))+\frac{\partial \psi^{l}}{\partial y^{k}}(x, f(x)) \partial_{i} f^{k}(x)
\end{aligned}
$$

We may introduce new coordinates $\left(x^{i}, y^{k}, y_{i}^{k}\right)$ transforming like:

$$
\bar{y}_{r}^{l} \partial_{i} \varphi^{r}(x)=\frac{\partial \psi^{l}}{\partial x^{i}}(x, y)+\frac{\partial \psi^{l}}{\partial y^{k}}(x, y) y_{i}^{k} .
$$

We shall denote by $J_{q}(\mathcal{E})$ the q-jet bundle of $\mathcal{E}$ with local coordinates

$$
\left(x^{i}, y^{k}, y_{i}^{k}, y_{i j}^{k}, \cdots\right)=\left(x, y_{q}\right)
$$

called jet coordinates and sections

$$
\begin{aligned}
& f_{q}:(x) \rightarrow\left(x, f^{k}(x), f_{i}^{k}(x), f_{i j}^{k}(x), \cdots\right) \\
& =\left(x, f_{q}(x)\right)
\end{aligned}
$$

transforming like the sections

$$
\begin{aligned}
& j_{q}(f):(x) \rightarrow\left(x, f^{k}(x), \partial_{i} f^{k}(x), \partial_{i j} f^{k}(x), \cdots\right) \\
& =\left(x, j_{q}(f)(x)\right)
\end{aligned}
$$

where both $f_{q}$ and $j_{q}(f)$ are over the section $f$ of $\mathcal{E}$. Of course $J_{q}(\mathcal{E})$ is a fibered manifold over $X$ with projection $\pi_{q}$ while $J_{q+r}(\mathcal{E})$ is a fibered manifold over $J_{q}(\mathcal{E})$ with projection $\pi_{q}^{q+r}, \forall r \geq 0$.

DEFINITION 1.1: A (nonlinear) system of order $q$ on $\mathcal{E}$ is a fibered submanifold $\mathcal{R}_{q} \subset J_{q}(\mathcal{E})$ and a solution of $\mathcal{R}_{q}$ is a section $f$ of $\mathcal{E}$ such that $j_{q}(f)$ is a section of $\mathcal{R}_{q}$.

DEFINITION 1.2: When the changes of coordinates have the linear form $\bar{x}=\varphi(x), \bar{y}=A(x) y$, we say that $\mathcal{E}$ is a vector bundle over $X$. Vector bundles will be denoted by capital letters $C, E, F$ and will have sections denoted by $\xi, \eta, \zeta$. In particular, we shall denote as usual by $T=T(X)$ the tangent bundle of $X$, by $T^{*}=T^{*}(X)$ the cotangent bundle, by $\wedge^{r} T^{*}$ the bundle of $r$-forms and by $S_{q} T^{*}$ the bundle of $q$ symmetric covariant tensors. When the changes of coordinates have the form

$$
\bar{x}=\varphi(x), \bar{y}=A(x) y+B(x)
$$

we say that $\mathcal{E}$ is an affine bundle over $X$ and we define the associated vector bundle $E$ over $X$ by the local coordinates $(x, v)$ changing like $\bar{X}=\varphi(x)$, $\bar{v}=A(x) v$. Finally, If $\mathcal{E}=X \times X$, we shall denote by $\Pi_{q}=\Pi_{q}(X, X)$ the open subfibered manifold of $J_{q}(X \times X)$ defined independently of the coordinate system by $\operatorname{det}\left(y_{i}^{k}\right) \neq 0$ with source projection

$$
\alpha_{q}: \Pi_{q} \rightarrow X:\left(x, y_{q}\right) \rightarrow(x)
$$

and target projection

$$
\beta_{q}: \Pi_{q} \rightarrow X:\left(x, y_{q}\right) \rightarrow(y) \text {. }
$$

DEFINITION 1.3: If the tangent bundle $T(\mathcal{E})$ has local coordinates $(x, y, u, v)$ changing like

$$
\begin{aligned}
& \bar{u}^{j}=\partial_{i} \varphi^{j}(x) u^{i}, \\
& \bar{v}^{l}=\frac{\partial \psi^{l}}{\partial x^{i}}(x, y) u^{i}+\frac{\partial \psi^{l}}{\partial y^{k}}(x, y) v^{k},
\end{aligned}
$$

we may introduce the vertical bundle $V(\mathcal{E}) \subset T(\mathcal{E})$ as a vector bundle over $\mathcal{E}$ with local coordinates $(x, y, v)$ obtained by setting $u=0$ and changes

$$
\bar{v}^{l}=\frac{\partial \psi^{l}}{\partial y^{k}}(x, y) v^{k} .
$$

Of course, when $\mathcal{E}$ is an affine bundle over $X$ with associated vector bundle $E$ over $X$, we have $V(\mathcal{E})=\mathcal{E} \times_{X} E$.

For a later use, if $\mathcal{E}$ is a fibered manifold over $X$ and $f$ is a section of $\mathcal{E}$, we denote by $f^{-1}(V(\mathcal{E}))$ the reciprocal image of $V(\mathcal{E})$ by $f$ as the vector bundle over $X$ obtained when replacing $(x, y, v)$ by $(x, f(x), v)$ in each chart. A similar construction may also be done for any affine bundle over $\mathcal{E}$.

We now recall a few basic geometric concepts that will be constantly used through this paper. First of all, if $\xi, \eta \in T$, we define their bracket $[\xi, \eta] \in T$ by the local formula

$$
([\xi, \eta])^{i}(x)=\xi^{r}(x) \partial_{r} \eta^{i}(x)-\eta^{s}(x) \partial_{s} \xi^{i}(x)
$$

leading to the Jacobi identity

$$
\begin{aligned}
& {[\xi,[\eta, \zeta]]+[\eta,[\zeta, \xi]]+[\zeta,[\xi, \eta]]=0,} \\
& \forall \xi, \eta, \zeta \in T
\end{aligned}
$$

allowing to define a Lie algebra and to the useful formula

$$
[T(f)(\xi), T(f)(\eta)]=T(f)([\xi, \eta])
$$

where $T(f): T(X) \rightarrow T(Y)$ is the tangent mapping of a map $f: X \rightarrow Y$. 
When $I=\left\{i_{1}<\cdots<i_{r}\right\}$ is a multi-index, we may set

$$
d x^{I}=d x^{i_{1}} \wedge \cdots \wedge d x
$$

for describing $\wedge^{r} T^{* i_{r}}$ by means of a basis and introduce the exterior derivative

$$
\begin{aligned}
& d: \wedge^{r} T^{*} \rightarrow \wedge^{r+1} T^{*}: \omega=\omega_{I} d x^{I} \rightarrow d \omega \\
& =\partial_{i} \omega_{I} d x^{i} \wedge d x^{I}
\end{aligned}
$$

with $d^{2}=d \circ d \equiv 0$ in the Poincaré sequence:

$$
\wedge^{0} T^{*} \stackrel{d}{\rightarrow} \wedge^{1} T^{*} \stackrel{d}{\rightarrow} \wedge^{2} T^{*} \stackrel{d}{\rightarrow} \cdots \stackrel{d}{\rightarrow} \wedge^{n} T^{*} \rightarrow 0 .
$$

The Lie derivative of an $r$-form with respect to a vector field $\xi \in T$ is the linear first order operator $\mathcal{L}(\xi)$ linearly depending on $j_{1}(\xi)$ and uniquely defined by the following three properties:

1) $\mathcal{L}(\xi) f=\xi . f=\xi^{i} \partial_{i} f, \forall f \in \wedge^{0} T^{*}=C^{\infty}(X)$.

2) $(\xi) d=d \mathcal{L}(\xi)$.

3) $\mathcal{L}(\xi)(\alpha \wedge \beta)=(\mathcal{L}(\xi) \alpha) \wedge \beta+\alpha \wedge(\mathcal{L}(\xi) \beta)$, $\forall \alpha, \beta \in \wedge T^{*}$.

It can be proved that

$$
\mathcal{L}(\xi)=i(\xi) d+d i(\xi)
$$

where $i(\xi)$ is the interior multiplication

$$
(i(\xi) \omega)_{i_{1} \cdots i_{r}}=\xi^{i} \omega_{i i_{1} \cdots i_{r}}
$$

and that

$$
\begin{aligned}
& {[\mathcal{L}(\xi), \mathcal{L}(\eta)]=\mathcal{L}(\xi) \circ \mathcal{L}(\eta)-\mathcal{L}(\eta) \circ \mathcal{L}(\xi)} \\
& =\mathcal{L}([\xi, \eta]), \forall \xi, \eta \in T .
\end{aligned}
$$

We now turn to group theory and start with two basic definitions:

Let $G$ be a Lie group, that is a manifold with local coordinates $\left(a^{\tau}\right)$ for $\tau=1, \cdots, p=\operatorname{dim}(G)$ called parameters, a composition

$$
G \times G \rightarrow G:(a, b) \rightarrow a b,
$$

an inverse $G \rightarrow G: a \rightarrow a^{-1}$ and an identity $e \in G$ satisfying:

$$
\begin{aligned}
& (a b) c=a(b c)=a b c, a a^{-1}=a^{-1} a=e, \\
& a e=e a=a \quad \forall a, b, c \in G .
\end{aligned}
$$

DEFINITION 1.4: $G$ is said to act on $X$ if there is a map $X \times G \rightarrow X:(x, a) \rightarrow y=a x=f(x, a)$ such that $(a b) x=a(b x)=a b x, \forall a, b \in G, \forall x \in X$ and we shall say that we have a Lie group of transformations of $X$. In order to simplify the notations, we shall use global notations even if only local actions are existing. It is well known that the action of $G$ onto itself allows to introduce a purely algebraic bracket on its Lie algebra $\mathcal{G}=T_{e}(G)$.
DEFINITION 1.5: A Lie pseudogroup of transformations $\Gamma \subset \operatorname{aut}(X)$ is a group of transformations solutions of a system of OD or PD equations such that, if $y=f(x)$ and $z=g(y)$ are two solutions, called finite transformations, that can be composed, then

$$
z=g \circ f(x)=h(x)
$$

and

$$
x=f^{-1}(y)=g(y)
$$

are also solutions while $y=x$ is the identity solution denoted by $i d=i d_{X}$ and we shall set $i d_{q}=j_{q}(i d)$. In all the sequel we shall suppose that $\Gamma$ is transitive that is

$$
\forall x, y \in X, \exists f \in \Gamma, y=f(x) .
$$

It becomes clear that Lie groups of transformations are particular cases of Lie pseudogroups of transformations as the system defining the finite transformations can be obtained by eliminating the parameters among the equations

$$
y_{q}=j_{q}(f)(x, a)
$$

when $q$ is large enough. The underlying system may be nonlinear and of high order. Looking for transformations "close" to the identity, that is setting $y=x+t \xi(x)+\cdots$ when $t \ll 1$ is a small constant parameter and passing to the limit $t \rightarrow 0$, we may linearize the above nonlinear system of finite Lie equations in order to obtain a linear system of infinitesimal Lie equations of the same order for vector fields. Such a system has the property that, if $\xi, \eta$ are two solutions, then $[\xi, \eta]$ is also a solution. Accordingly, the set $\Theta \subset T$ of solutions of this new system satisfies $[\Theta, \Theta] \subset \Theta$ and can therefore be considered as the Lie algebra of $\Gamma$.

EXAMPLE 1.6: While the affine transformations $y=a x+b$ are solutions of the second order linear system $y_{x x}=0$, the projective transformations

$$
y=(a x+b) /(c x+d)
$$

are solutions of the third order nonlinear system

$$
\Psi \equiv\left(y_{x x x} / y_{x}\right)-\frac{3}{2}\left(y_{x x} / y_{x}\right)^{2}=0 .
$$

The sections of the corresponding linearized systems are respectively satisfying $\xi_{x x}=0$ and $\xi_{x x x}=0$. The generating differential invariant $\Phi \equiv y_{x x} / y_{x}$ of the affine case transforms like

$$
u=\bar{u} \partial_{x} f+\left(\partial_{x x} f / \partial_{x} f\right)
$$

when $\bar{x}=f(x)$ while $\Psi$ transforms like

$$
v=\bar{v}\left(\partial_{x} f\right)^{2}+\left(\partial_{x x x} f / \partial_{x} f\right)-\frac{3}{2}\left(\partial_{x x} f / \partial_{x} f\right)^{2} .
$$


We now sketch the discovery of Vessiot $[8,9]$ still not known today after more than a century for reasons which are not scientific at all. Roughly, a Lie pseudogroup $\Gamma \subset \operatorname{aut}(X)$ is made by finite transformations $y=f(x)$ solutions of a (possibly nonlinear) system $\mathcal{R}_{q} \subset \Pi_{q}$ while the infinitesimal transformations $\xi \in \Theta$ are solutions of the linearized system

$$
R_{q}=i d_{q}^{-1}\left(V\left(\mathcal{R}_{q}\right)\right) \subset J_{q}(T)
$$

as we have

$$
T=i d^{-1}(V(X \times X)) .
$$

When $\Gamma$ is transitive, there is a canonical epimorphism $\pi_{0}^{q}: R_{q} \rightarrow T$. Also, as changes of source $x$ commute with changes of target $y$, they exchange between themselves any generating set of differential invariants $\left\{\Phi^{\tau}\left(y_{q}\right)\right\}$ as in the previous example. Then one can introduce a natural bundle $\mathcal{F}$ over $X$, also called bundle of geomeric objects, by patching changes of coordinates of the form

$$
\bar{x}=f(x), \bar{u}=\lambda\left(u, j_{q}(f(x))\right.
$$

thus obtained. A section $\omega$ of $\mathcal{F}$ is called a geometric object or structure on $X$ and transforms like

$$
\bar{\omega}(f(x))=\lambda\left(\omega(x), j_{q}(f)(x)\right)
$$

or simply $\bar{\omega}=j_{q}(f)^{-1}(\omega)$. This is a way to generalize vectors and tensors $(q=1)$ or even connections $(q=2)$. As a byproduct we have

$$
\Gamma=\left\{f \in \operatorname{aut}(X) \mid j_{q}(f)^{-1}(\omega)=\omega\right\}
$$

and we may say that $\Gamma$ preserves $\omega$. Replacing $j_{q}(f)$ by $f_{q}$, we also obtain

$$
\mathcal{R}_{q}=\left\{f_{q} \in \Pi_{q} \mid f_{q}^{-1}(\omega)=\omega\right\} .
$$

Coming back to the infinitesimal point of view and setting

$$
f_{t}=\exp (t \xi) \in \operatorname{aut}(X), \forall \xi \in T,
$$

we may define the ordinary Lie derivative with value in the vector bundle $F_{0}=\omega^{-1}(V(\mathcal{F}))$ by the formula:

$$
\begin{aligned}
\mathcal{D} \xi & =\mathcal{L}(\xi) \omega=\left.\frac{\mathrm{d}}{\mathrm{d} t} j_{q}(f)_{t}^{-1}(\omega)\right|_{t=0} \Rightarrow \Theta \\
& =\{\xi \in T \mid \mathcal{L}(\xi) \omega=0\}
\end{aligned}
$$

and we say that $\mathcal{D}$ is a Lie operator because $\mathcal{D} \xi=0, \mathcal{D} \eta=0 \Rightarrow \mathcal{D}[\xi, \eta]=0$ as we already saw.

Differentiating $r$ times the equations of $R_{q}$ that only depend on $j_{1}(\omega)$, we may obtain the $r$ prolongation

$$
R_{q+r}=J_{r}\left(R_{q}\right) \cap J_{q+r}(T) \subset J_{r}\left(J_{q}(T)\right) .
$$

The problem is then to know under what conditions on $\omega$ all the equations of order $q+r$ are obtained by $r$ prolongations only, $\forall r \geq 0$ or, equivalently, $R_{q}$ is formally integrable (FI). The solution, found by Vessiot, has been to exhibit another natural vector bundle $\mathcal{F}_{1}$ with local coordinates $(x, u, v)$ over $\mathcal{F}$ with local coordinates $(x, u)$ and to prove that an equivariant section $c: \mathcal{F} \rightarrow \mathcal{F}_{1}:(x, u) \rightarrow(x, u, v=c(u))$ only depends on a finite number of constants called structure constants. The integrability conditions (IC) of $R_{q}$, called Vessiot structure equations, are of the form $I\left(j_{1}(\omega)\right)=c(\omega)$ and are invariant under any change of source.

We provide in a self-contained way and parallel manners the following five striking examples which are among the best nontrivial ones we know and invite the reader to imagine at this stage any possible link that could exist between them (A few specific definitions will be given later on).

EXAMPLE 1.7: Coming back to the last example, we show that Vessiot structure equations may even exist when $n=1$. For this, if $\gamma$ is the geometric object of the affine group $y=a x+b$ and $0 \neq \alpha=\alpha(x) \mathrm{d} x \in T^{*}$ is a 1 -form, we consider the object $\omega=(\alpha, \gamma)$ and get at once the two second order Medolaghi equations:

$$
\begin{aligned}
& \mathcal{L}(\xi) \alpha \equiv \alpha \partial_{x} \xi+\xi \partial_{x} \alpha=0, \\
& \mathcal{L}(\xi) \gamma \equiv \partial_{x x} \xi+\gamma \partial_{x} \xi+\xi \partial_{x} \gamma=0
\end{aligned}
$$

Differentiating the first equation and substituting the second, we get the zero order equation:

$$
\begin{aligned}
& \xi\left(\alpha \partial_{x x} \alpha-2\left(\partial_{x} \alpha\right)^{2}+\alpha \gamma \partial_{x} \alpha-\alpha^{2} \partial_{x} \gamma\right) \\
& =0 \Leftrightarrow \xi \partial_{x}\left(\frac{\partial_{x} \alpha}{\alpha^{2}}-\frac{\gamma}{\alpha}\right)=0
\end{aligned}
$$

and the Vessiot structure equation $\partial_{x} \alpha-\gamma \alpha=c \alpha^{2}$. Alternatively, setting

$$
\beta=-1 / \alpha \in T,
$$

we get

$$
\partial_{x} \beta+\gamma \beta=c
$$

With

$$
\alpha=1, \beta=-1, \gamma=0 \Rightarrow c=0
$$

we get the translation subgroup $y=x+b$ while, with

$$
\alpha=1 / x, \beta=-x, \gamma=0 \Rightarrow c=-1
$$

we get the dilatation subgroup $y=a x$. Similarly, if $v$ is the geometric object of the projective group and we consider the new geometric object $\omega=(\gamma, v)$, we get the only Vessiot structure equation

$$
\partial_{x} \gamma-\frac{1}{2} \gamma^{2}-v=0
$$


without any structure constant.

EXAMPLE 1.8: (Principal homogeneous structure) When $\Gamma$ is the Lie group of transformations made by the constant translations $y^{i}=x^{i}+a^{i}$ for $i=1, \cdots, n$ of a manifold $X$ with $\operatorname{dim}(X)=n$, the characteristic object invariant by $\Gamma$ is a family

$$
\omega=\left(\omega^{\tau}=\omega_{i}^{\tau} \mathrm{d} x^{i}\right) \in T^{*} \times_{X} \cdots \times_{X} T^{*}
$$

of $n 1$-forms with $\operatorname{det}(\omega) \neq 0$ in such a way that

$$
\Gamma=\left\{f \in \operatorname{aut}(X) \mid j_{1}(f)^{-1}(\omega)=\omega\right\}
$$

where $\operatorname{aut}(X)$ denotes the pseudogroup of local diffeomorphisms of $X, j_{q}(f)$ denotes the derivatives of $f$ up to order $q$ and $j_{1}(f)$ acts in the usual way on covariant tensors. For any vector field $\xi \in T=T(X)$ the tangent bundle to $X$, introducing the standard Lie derivative $\mathcal{L}(\xi)$ of forms with respect to $\xi$, we may consider the $n^{2}$ first order Medolaghi equations:

$$
\Omega_{i}^{\tau} \equiv(\mathcal{L}(\xi) \omega)_{i}^{\tau} \equiv \omega_{r}^{\tau}(x) \partial_{i} \xi^{r}+\xi^{r} \partial_{r} \omega_{i}^{\tau}(x)=0 .
$$

The particular situation is found with the special choice $\omega=\left(d x^{i}\right)$ that leads to the involutive system $\partial_{i} \xi^{k}=0$. Introducing the inverse matrix $\alpha=\left(\alpha_{\tau}^{i}\right)=\omega^{-1}$, the above equations amount to the bracket relations $\left[\xi, \alpha_{\tau}\right]=0$ and, using crossed derivatives on the solved form

$$
\partial_{i} \xi^{k}+\xi^{r} \alpha_{\tau}^{k}(x) \partial_{r} \omega_{i}^{\tau}(x)=0,
$$

we obtain the $n^{2}(n-1) / 2$ zero order equations:

$$
\xi^{r} \partial_{r}\left(\alpha_{\rho}^{i}(x) \alpha_{\sigma}^{j}(x)\left(\partial_{i} \omega_{j}^{\tau}(x)-\partial_{j} \omega_{i}^{\tau}(x)\right)\right)=0 .
$$

The integrability conditions (IC), that is the conditions under which these equations do not bring new equations, are thus the $n^{2}(n-1) / 2$ Vessiot structure equations:

$$
\partial_{i} \omega_{j}^{\tau}(x)-\partial_{j} \omega_{i}^{\tau}(x)=c_{\rho \sigma}^{\tau} \omega_{i}^{\rho}(x) \omega_{j}^{\sigma}(x)
$$

with $n^{2}(n-1) / 2$ structure constants $c=\left(c_{\rho \sigma}^{\tau}=-c_{\sigma \rho}^{\tau}\right)$.

When $X=G$, these equations can be identified with the Maurer-Cartan equations (MC) existing in the theory of Lie groups, on the condition to change the sign of the structure constants involved because we have

$$
\left[\alpha_{\rho}, \alpha_{\sigma}\right]=-c_{\rho \sigma}^{\tau} \alpha_{\tau} .
$$

Writing these equations in the form of the exterior system $d \omega^{\tau}=c_{\rho \sigma}^{\tau} \omega^{\rho} \wedge \omega$ and closing this system by applying once more the exterior derivative $d$, we obtain the quadratic IC:

$$
c_{\mu \rho}^{\lambda} c_{\sigma \tau}^{\mu}+c_{\mu \sigma}^{\lambda} c_{\tau \rho}^{\mu}+c_{\mu \tau}^{\lambda} c_{\rho \sigma}^{\mu}=0
$$

also called Jacobi relations $J(c)=0$.

EXAMPLE 1.9: (Riemann structure) If

$$
\omega=\left(\omega_{i j}=\omega_{j i}\right) \in S_{2} T^{*}
$$

is a metric on a manifold $X$ with $\operatorname{dim}(X)=n$ such that $\operatorname{det}(\omega) \neq 0$, the Lie pseudogroup of transformations preserving $\omega$ is

$$
\Gamma=\left\{f \in \operatorname{aut}(X) \mid j_{1}(f)^{-1}(\omega)=\omega\right\}
$$

and is a Lie group with a maximum number of $n(n+1) / 2$ parameters. A special metric could be the Euclidean metric when $n=1,2,3$ as in elasticity theory or the Minkowski metric when $n=4$ as in special relativity [10]. The first order Medolaghi equations:

$$
\begin{aligned}
\Omega_{i j} & \equiv(\mathcal{L}(\xi) \omega)_{i j} \\
& \equiv \omega_{r j}(x) \partial_{i} \xi^{r}+\omega_{i r}(x) \partial_{j} \xi^{r}+\xi^{r} \partial_{r} \omega_{i j}(x)=0
\end{aligned}
$$

are also called classical Killing equations for historical reasons. The main problem is that this system is not involutive unless we prolong it to order two by differentiating once the equations. For such a purpose, introducing $\omega^{-1}=\left(\omega^{i j}\right)$ as usual, we may define the Christoffel symbols:

$$
\begin{aligned}
\gamma_{i j}^{k}(x) & =\frac{1}{2} \omega^{k r}(x)\left(\partial_{i} \omega_{r j}(x)+\partial_{j} \omega_{r i}(x)-\partial_{r} \omega_{i j}(x)\right) \\
& =\gamma_{j i}^{k}(x) .
\end{aligned}
$$

This is a new geometric object of order 2 providing the Levi-Civita isomorphism $j_{1}(\omega)=(\omega, \partial \omega) \simeq(\omega, \gamma)$ of affine bundles and allowing to obtain the second order Medolaghi equations:

$$
\begin{aligned}
\Gamma_{i j}^{k} & \equiv(\mathcal{L}(\xi) \gamma)_{i j}^{k} \equiv \partial_{i j} \xi^{k}+\gamma_{r j}^{k}(x) \partial_{i} \xi^{r}+\gamma_{i r}^{k}(x) \partial_{j} \xi^{r} \\
& -\gamma_{i j}^{r}(x) \partial_{r} \xi^{k}+\xi^{r} \partial_{r} \gamma_{i j}^{k}(x)=0
\end{aligned}
$$

Surprisingly, the following expression called Riemann tensor:

$$
\begin{aligned}
\rho_{l i j}^{k}(x) & \equiv \partial_{i} \gamma_{l j}^{k}(x)-\partial_{j} \gamma_{l i}^{k}(x) \\
& +\gamma_{l j}^{r}(x) \gamma_{r i}^{k}(x)-\gamma_{l i}^{r}(x) \gamma_{r j}^{k}(x)
\end{aligned}
$$

is still a first order geometric object and even a 4 -tensor with $n^{2}\left(n^{2}-1\right) / 12$ independent components satisfying the purely algebraic relations:

$$
\rho_{l i j}^{k}+\rho_{i j l}^{k}+\rho_{j l i}^{k}=0, \quad \omega_{r l} \rho_{k i j}^{l}+\omega_{k r} \rho_{l i j}^{r}=0 .
$$

Accordingly, the IC must express that the new first order equations $R_{l i j}^{k} \equiv(\mathcal{L}(\xi) \rho)_{l i j}^{k}=0$ are only linear combinations of the previous ones and we get the Vessiot structure equations:

$$
\rho_{l i j}^{k}(x)=c\left(\delta_{i}^{k} \omega_{l j}(x)-\delta_{j}^{k} \omega_{l i}(x)\right)
$$

with the only structure constant $c$ describing the constant Riemannian curvature condition of Eisenhart 
$[11,12]$. One can proceed similarly for the conformal Killing system $\mathcal{L}(\xi) \omega=A(x) \omega$ and obtain that the Weyl tensor must vanish, without any structure constant [12].

EXAMPLE 1.10: (Contact structure) We only treat the case $\operatorname{dim}(X)=3$ as the case $\operatorname{dim}(X)=2 p+1$ needs much more work [6]. Let us consider the so-called contact 1 -form $\alpha=d x^{1}-x^{3} d x^{2}$ and consider the Lie pseudogroup $\Gamma \subset \operatorname{aut}(X)$ of (local) transformations preserving $\alpha$ up to a function factor, that is

$$
\Gamma=\left\{f \in \operatorname{aut}(X) \mid j_{1}(f)^{-1}(\alpha)=\rho \alpha\right\}
$$

where again $j_{q}(f)$ is a symbolic way for writing out the derivatives of $f$ up to order $q$ and $\alpha$ transforms like a 1 -covariant tensor. It may be tempting to look for a kind of "object" the invariance of which should characterize $\Gamma$. Introducing the exterior derivative $d \alpha=d x^{2} \wedge d x^{3}$ as a 2 -form, we obtain the volume 3 -form $\alpha \wedge d \alpha=d x^{1} \wedge d x^{2} \wedge d x^{3}$. As it is well known that the exterior derivative commutes with any diffeomorphism, we obtain sucessively:

$$
\begin{aligned}
& j_{1}(f)^{-1}(d \alpha)=d\left(j_{1}(f)^{-1}(\alpha)\right)=d(\rho \alpha) \\
& =\rho d \alpha+d \rho \wedge \alpha \Rightarrow j_{1}(f)^{-1}(\alpha \wedge d \alpha) \\
& =\rho^{2}(\alpha \wedge d \alpha) .
\end{aligned}
$$

As the volume 3 -form $\alpha \wedge d \alpha$ transforms through a division by the Jacobian determinant

$$
\Delta=\partial\left(f^{1}, f^{2}, f^{3}\right) / \partial\left(x^{1}, x^{2}, x^{3}\right) \neq 0
$$

of the transformation $y=f(x)$ with inverse

$$
x=f^{-1}(y)=g(y),
$$

the desired object is thus no longer a 1-form but a 1form density $\omega=\left(\omega_{1}, \omega_{2}, \omega_{3}\right)$ transforming like a 1form but up to a division by the square root of the Jacobian determinant. It follows that the infinitesimal contact transformations are vector fields $\xi \in T=T(X)$ the tangent bundle of $X$, satisfying the 3 so-called first order Medolaghi equations:

$$
\begin{aligned}
\Omega_{i} & \equiv(\mathcal{L}(\xi) \omega)_{i} \\
& \equiv \omega_{r}(x) \partial_{i} \xi^{r}-(1 / 2) \omega_{i}(x) \partial_{r} \xi^{r}+\xi^{r} \partial_{r} \omega_{i}(x)=0
\end{aligned} .
$$

When $\omega=\left(1,-x^{3}, 0\right)$, we obtain the special involutive system:

$$
\begin{aligned}
& \partial_{3} \xi^{3}+\partial_{2} \xi^{2}+2 x^{3} \partial_{1} \xi^{2}-\partial_{1} \xi^{1}=0 \\
& \partial_{3} \xi^{1}-x^{3} \partial_{3} \xi^{2}=0, \\
& \partial_{2} \xi^{1}-x^{3} \partial_{2} \xi^{2}+x^{3} \partial_{1} \xi^{1}-\left(x^{3}\right)^{2} \partial_{1} \xi^{2}-\xi^{3}=0
\end{aligned}
$$

with 2 equations of class 3 and 1 equation of class 2 (see later on for a precise definition) and thus only $1 \mathrm{com}$ - patibility conditions (CC) for the second members.

For an arbitrary $\omega$, we may ask about the differential conditions on $\omega$ such that all the equations of order $r+1$ are only obtained by differentiating $r$ times the first order equations, exactly like in the special situation just considered where the system is involutive. We notice that, in a symbolic way, $\omega \wedge d \omega$ is now a scalar $c(x)$ providing the zero order equation $\xi^{r} \partial_{r} c(x)=0$ and the condition is $c(x)=c=c s t$. The integrability condition (IC) is the Vessiot structure equation:

$$
\begin{aligned}
& \omega_{1}\left(\partial_{2} \omega_{3}-\partial_{3} \omega_{2}\right)+\omega_{2}\left(\partial_{3} \omega_{1}-\partial_{1} \omega_{3}\right) \\
& +\omega_{3}\left(\partial_{1} \omega_{2}-\partial_{2} \omega_{1}\right)=c
\end{aligned}
$$

involving the only structure constant $c$.

For $\omega=\left(1,-x^{3}, 0\right)$, we get $c=1$. If we choose $\bar{\omega}=(1,0,0)$ leading to $\bar{c}=0$, we may define

$$
\bar{\Gamma}=\left\{f \in \operatorname{aut}(X) \mid j_{1}(f)^{-1}(\bar{\omega})=\bar{\omega}\right\}
$$

with infinitesimal transformations satisfying the involutive system:

$$
\partial_{3} \xi^{3}+\partial_{2} \xi^{2}-\partial_{1} \xi^{1}=0, \partial_{3} \xi^{1}=0, \partial_{2} \xi^{1}=0
$$

with again 2 equations of class 3 and 1 equation of class 2.

EXAMPLE 1.11: (Unimodular contact structure) With similar notations, let us again set

$$
\alpha=d x^{1}-x^{3} d x^{2} \Rightarrow d \alpha=d x^{2} \wedge d x^{3}
$$

but let us now consider the new Lie pseudogroup of transformations preserving $\alpha$ and thus $d \alpha$ too, that is preserving the mixed object

$$
\omega=(\alpha, \beta) \in T^{*} \times_{X} \wedge{ }^{2} T^{*}
$$

made up by a 1 -form $\alpha$ and a 2 -form $\beta$ with

$$
\gamma=\alpha \wedge \beta \neq 0 \text { and } d \alpha=\beta \Rightarrow d \beta=0 .
$$

Then $\Gamma$ is a Lie subpseudogroup of the one just considered in the previous example and the corresponding infinitesimal transformations now satisfy the involutive system:

$$
\begin{aligned}
& \partial_{1} \xi^{1}=0, \partial_{1} \xi^{2}=0, \partial_{1} \xi^{3}=0, \partial_{2} \xi^{1}+x^{3} \partial_{3} \xi^{3}-\xi^{3}=0, \\
& \partial_{2} \xi^{2}+\partial_{3} \xi^{3}=0, \partial_{3} \xi^{1}-x^{3} \partial_{3} \xi^{2}=0
\end{aligned}
$$

with 3 equations of class 3, 2 equations of class 2 and 1 equation of class 1 if we exchange $x^{1}$ with $x^{3}$, a result leading now to $4 \mathrm{CC}$.

More generally, when $\omega=(\alpha, \beta)$ where $\alpha$ is a 1 form and $\beta$ is a 2 -form satifying $\alpha \wedge \beta \neq 0$, we may study the same problem as before for the general system

$$
\mathcal{L}(\xi) \alpha=0, \mathcal{L}(\xi) \beta=0 .
$$

We let the reader provide the details of the tedious computation involved as it is at this point that computer 
algebra may be used [13]. The result, not evident at first sight, is that the 2-form $d \alpha$ must be proportional to the 2-form $\beta$, that is $d \alpha=c^{\prime}(x) \beta$ and thus

$$
\alpha \wedge d \alpha=c^{\prime}(x) \alpha \wedge \beta \text {. }
$$

As $\alpha \wedge \beta \neq 0$, we must have $c^{\prime}(x)=c^{\prime}=c s t$ and thus $d \alpha=c^{\prime} \beta$. Similarly, we get $d \beta=c^{\prime \prime} \alpha \wedge \beta$ and obtain finally the 4 Vessiot structure equations

$$
d \alpha=c^{\prime} \beta, d \beta=c^{\prime \prime} \alpha \wedge \beta
$$

involving 2 structure constants $c=\left(c^{\prime}, c^{\prime \prime}\right)$. Contrary to the previous situation (but like in the Riemann case!) we notice that we have now 2 structure equations not containing any constant (called first kind by Vessiot) and 2 structure equations with the same number of different constants (called second kind by Vessiot), namely

$$
\alpha \wedge d \alpha=c^{\prime} \alpha \wedge \beta, d \beta=c^{\prime \prime} \alpha \wedge \beta .
$$

Finally, closing this system by taking once more the exterior derivative, we get

$$
0=d^{2} \alpha=c^{\prime} d \beta=c^{\prime} c^{\prime \prime} \alpha \wedge \beta
$$

and thus the unexpected purely algebraic Jacobi condition $c^{\prime} C^{\prime \prime}=0$. For the special choice

$$
\omega=\left(d x^{1}-x^{3} d x^{2}, d x^{2} \wedge d x^{3}\right)
$$

we get $c=(1,0)$, for the second special choice

$$
\bar{\omega}=\left(d x^{1}, d x^{2} \wedge d x^{3}\right)
$$

we get $\bar{c}=(0,0)$ and for the third special choice

$$
\overline{\bar{\omega}}=\left(\left(1 / x^{1}\right) d x^{1}, x^{1} d x^{2} \wedge d x^{3}\right)
$$

we get $\overline{\bar{C}}=(0,1)$.

FIRST FUNDAMENTAL RESULT: Comparing the various Vessiot structure equations containing structure constants that we have just presented and that we recall below in a symbolic way, we notice that these structure constants are absolutely on equal footing though they have in general nothing to do with any Lie algebra.

$$
\begin{aligned}
& \left\{\begin{array}{l}
\partial \alpha-\gamma \alpha=c \alpha^{2} \\
\partial \beta+\gamma \beta=c
\end{array}\right. \\
& \partial \omega-\partial \omega=c \omega \omega \\
& \partial \gamma-\partial \gamma+\gamma \gamma-\gamma \gamma=c(\delta \omega-\delta \omega) \\
& \omega \wedge(\partial \omega-\partial \omega)=c
\end{aligned}
$$

Accordingly, the fact that the ones appearing in the $\mathrm{MC}$ equations are related to a Lie algebra is a coincidence and the Cartan structure equations have nothing to do with the Vessiot structure equations. Also, as their factors are either constant, linear or quadratic, any identification of the quadratic terms appearing in the Riemann tensor with the quadratic terms appearing in the MC equations is definitively not correct [7]. We also understand why the torsion is automatically combined with curvature in the Cartan structure equations but totally absent from the Vessiot structure equations, even though the underlying group (translations + rotations) is the same.

HISTORICAL REMARK 1.12: Despite the prophetic comments of the italian mathematician Ugo Amaldi in 1909 [12], it has been a pity that Cartan deliberately ignored the work of Vessiot at the beginning of the last century and that the things did not improve afterwards in the eighties with Spencer and coworkers (Compare MR 720863 (85 m: 12004) and MR 954613 (90e: 58166)).

\section{Second Part: The Janet and Spencer Sequences}

Let $\mu=\left(\mu_{1}, \cdots, \mu_{n}\right)$ be a multi-index with length

$$
|\mu|=\mu_{1}+\cdots+\mu_{n}
$$

class $i$ if

$$
\mu_{1}=\cdots=\mu_{i-1}=0, \mu_{i} \neq 0
$$

and

$$
\mu+1_{i}=\left(\mu_{1}, \cdots, \mu_{i-1}, \mu_{i}+1, \mu_{i+1}, \cdots, \mu_{n}\right) .
$$

We set

$$
y_{q}=\left\{y_{\mu}^{k}|1 \leq k \leq m, 0 \leq| \mu \mid \leq q\right\}
$$

with $y_{\mu}^{k}=y^{k}$ when $|\mu|=0$. If $E$ is a vector bundle over $X$ with local coordinates $(x, y)$ and $J_{q}(E)$ is the $q$-jet bundle of $E$ with local coordinates $\left(x, y_{q}\right)$, the Spencer operator just allows to distinguish a section $\xi_{q}$ from a section $j_{q}(\xi)$ by introducing a kind of "difference" through the operator

$$
D: J_{q+1}(E) \rightarrow T^{*} \otimes J_{q}(E): \xi_{q+1} \rightarrow j_{1}\left(\xi_{q}\right)-\xi_{q+1}
$$

with local components

$$
\left(\partial_{i} \xi^{k}(x)-\xi_{i}^{k}(x), \partial_{i} \xi_{j}^{k}(x)-\xi_{i j}^{k}(x), \cdots\right)
$$

and more generally

$$
\left(D \xi_{q+1}\right)_{\mu, i}^{k}(x)=\partial_{i} \xi_{\mu}^{k}(x)-\xi_{\mu+1_{i}}^{k}(x) .
$$

Minus the restriction of $D$ to the kernel $S_{q+1} T^{*} \otimes E$ of the canonical projection

$$
\pi_{q}^{q+1}: J_{q+1}(E) \rightarrow J_{q}(E)
$$

can be extended to the Spencer map 


$$
\delta: \wedge^{s} T^{*} \otimes S_{q+1} T^{*} \otimes E \rightarrow \wedge^{s+1} T^{*} \otimes S_{q} T^{*} \otimes E
$$

defined by

$$
(\delta \omega)_{\mu}^{k}=d x^{i} \wedge \omega_{\mu+1_{i}}^{k} .
$$

The kernel of $D$ is made by sections such that

$$
\xi_{q+1}=j_{1}\left(\xi_{q}\right)=j_{2}\left(\xi_{q-1}\right)=\cdots=j_{q+1}(\xi) .
$$

Finally, if $R_{q} \subset J_{q}(E)$ is a system of order $q$ on $E$ locally defined by linear equations

$$
\Phi^{\tau}\left(x, y_{q}\right) \equiv a_{k}^{\tau \mu}(x) y_{\mu}^{k}=0,
$$

the $r$-prolongation

$$
\begin{aligned}
R_{q+r} & =\rho_{r}\left(R_{q}\right) \\
& =J_{r}\left(R_{q}\right) \cap J_{q+r}(E) \subset J_{r}\left(J_{q}(E)\right)
\end{aligned}
$$

is locally defined when $r=1$ by the linear equations

$$
\begin{aligned}
& \Phi^{\tau}\left(x, y_{q}\right)=0, d_{i} \Phi^{\tau}\left(x, y_{q+1}\right) \\
& \equiv a_{k}^{\tau \mu}(x) y_{\mu+1_{i}}^{k}+\partial_{i} a_{k}^{\tau \mu}(x) y_{\mu}^{k}=0
\end{aligned}
$$

and has symbol

$$
g_{q+r}=R_{q+r} \cap S_{q+r} T^{*} \otimes E \subset J_{q+r}(E)
$$

locally defined by

$$
a_{k}^{\tau \mu}(x) \xi_{\mu+v}^{k}=0,|\mu|=q,|v|=r
$$

if one looks at the top order terms. If $\xi_{q+1} \in R_{q+1}$ is over $\xi_{q} \in R_{q}$, differentiating the identity

$$
a_{k}^{\tau \mu}(x) \xi_{\mu}^{k}(x) \equiv 0
$$

with respect to $x^{i}$ and substracting the identity

$$
a_{k}^{\tau \mu}(x) \xi_{\mu+1_{i}}^{k}(x)+\partial_{i} a_{k}^{\tau \mu}(x) \xi_{\mu}^{k}(x) \equiv 0,
$$

we obtain the identity

$$
a_{k}^{\tau \mu}(x)\left(\partial_{i} \xi_{\mu}^{k}(x)-\xi_{\mu+1_{i}}^{k}(x)\right) \equiv 0
$$

and thus the restriction $D: R_{q+1} \rightarrow T^{*} \otimes R_{q}$. This first order operator induces, up to sign, the purely algebraic monomorphism $0 \rightarrow g_{q+1} \stackrel{\delta}{\rightarrow} T^{*} \otimes g_{q}$ on the symbol level $[8,14]$. The Spencer operator has never been used in GR though an accelerometer in a rocket merely measures one of the components of the Spencer operator involving second order jets.

DEFINITION 2.1: $R_{q}$ is said to be formally integrable $(\mathrm{FI})$ when the restriction

$$
\pi_{q+r}^{q+r+1}: R_{q+r+1} \rightarrow R_{q+r}
$$

is an epimorphism $\forall r \geq 0$. In that case, the Spencer form $R_{q+1} \subset J_{1}\left(R_{q}\right)$ is a canonical equivalent formally integrable first order system on $R_{q}$ with no zero order equations.

DEFINITION 2.2: $R_{q}$ is said to be involutive when it is formally integrable and the symbol $g_{q}$ is involutive, that is all the sequences

$$
\cdots \stackrel{\delta}{\rightarrow} \wedge^{s} T^{*} \otimes g_{q+r} \stackrel{\delta}{\rightarrow} \cdots
$$

are exact $\forall 0 \leq s \leq n, \forall r \geq 0$. Equivalently, using a linear change of local coordinates if necessary, we may successively solve the maximum number $\beta_{q}^{n}, \beta_{q}^{n-1}, \cdots, \beta_{q}^{1}$ of equations with respect to the leading or principal jet coordinates of strict order $q$ and class $n, n-1, \cdots, 1$. Then $R_{q}$ is involutive if $R_{q+1}$ is obtained by only prolonging the $\beta_{q}^{i}$ equations of class $i$ with respect to $d_{1}, \cdots, d_{i}$ for $i=1, \cdots, n$. In that case, such a prolongation procedure allows to compute in a unique way the principal jets from the parametric other ones and may also be applied to nonlinear systems as well $[8,15]$.

When $R_{q}$ is involutive, the linear differential operator

$$
D: E \stackrel{j_{q}}{\rightarrow} J_{q}(E) \stackrel{\Phi}{\rightarrow} J_{q}(E) / R_{q}=F_{0}
$$

of order $q$ with space of solutions $\Theta \subset E$ is said to be involutive and one has the canonical linear Janet sequence [8]:

$$
0 \rightarrow \Theta \rightarrow E \stackrel{\mathcal{D}}{\rightarrow} F_{0} \stackrel{\mathcal{D}_{1}}{\rightarrow} F_{1} \stackrel{\mathcal{D}_{2}}{\rightarrow} \cdots \stackrel{\mathcal{D}_{n}}{\rightarrow} F_{n} \rightarrow 0
$$

with Janet bundles

$$
\begin{aligned}
& F_{r}= \\
& \wedge^{r} T^{*} \otimes J_{q}(E) /\left(\wedge^{r} T^{*} \otimes R_{q}+\delta\left(\wedge^{r-1} T^{*} \otimes S_{q+1} T^{*} \otimes E\right)\right) .
\end{aligned}
$$

Each operator $\mathcal{D}_{r+1}: F_{r} \rightarrow F_{r+1}$ is first order involutive as it is induced by

$$
\begin{aligned}
& D: \wedge^{r} T^{*} \otimes J_{q+1}(E) \rightarrow \wedge^{r+1} T^{*} \otimes J_{q}(E): \alpha \otimes \xi_{q+1} \\
& \rightarrow d \alpha \otimes \xi_{q}+(-1)^{r} \alpha \wedge D \xi_{q+1}
\end{aligned}
$$

and generates the compatibility conditions (CC) of the preceding one. As the Janet sequence can be cut at any place, the numbering of the Janet bundles has nothing to do with that of the Poincaré sequence, contrary to what many people believe in GR.

Similarly, we have the involutive first Spencer operator

$$
\begin{aligned}
D_{1}: C_{0} & =R_{q} \stackrel{j_{1}}{\rightarrow} J_{1}\left(R_{q}\right) \rightarrow J_{1}\left(R_{q}\right) / R_{q+1} \\
& \simeq T^{*} \otimes R_{q} / \delta\left(g_{q+1}\right)=C_{1}
\end{aligned}
$$

of order one induced by $D: R_{q+1} \rightarrow T^{*} \otimes R_{q}$. Introducing the Spencer bundles

$$
C_{r}=\wedge^{r} T^{*} \otimes R_{q} / \delta\left(\wedge^{r-1} T^{*} \otimes g_{q+1}\right),
$$


the first order involutive $(r+1)$ Spencer operator $D_{r+1}: C_{r} \rightarrow C_{r+1}$ is induced by

$$
D: \wedge^{r+1} T^{*} \otimes R_{q+1} \rightarrow \wedge^{r+1} T^{*} \otimes R_{q}
$$

and we obtain the canonical linear Spencer sequence $[8,14]$ :

$$
0 \rightarrow \Theta \stackrel{j_{q}}{\rightarrow} C_{0} \stackrel{D_{1}}{\rightarrow} C_{1} \stackrel{D_{2}}{\rightarrow} C_{2} \stackrel{D_{3}}{\rightarrow} \cdots \stackrel{D_{n}}{\rightarrow} C_{n} \rightarrow 0
$$

as the Janet sequence for the first order involutive system $R_{q+1} \subset J_{1}\left(R_{q}\right)$. Introducing the other Spencer bundles

$$
C_{r}(E)=\wedge^{r} T^{*} \otimes J_{q}(E) / \delta\left(\wedge^{r-1} T^{*} \otimes S_{q+1} T^{*} \otimes E\right)
$$

In this diagram, only depending on the linear differential operator $\mathcal{D}=\Phi \circ j_{q}$, the epimorhisms

$$
\Phi_{r}: C_{r}(E) \rightarrow F_{r} \text { for } 0 \leq r \leq n
$$

are induced by the canonical projection

$$
\Phi=\Phi_{0}: C_{0}(E)=J_{q}(E) \rightarrow J_{q}(E) / R_{q}=F_{0}
$$

if we start with the knowledge of $R_{q} \subset J_{q}(E)$ or from the knowledge of an epimorphism

$$
\Phi: J_{q}(E) \rightarrow F_{0}
$$

if we set $R_{q}=\operatorname{ker}(\Phi)$. In the theory of Lie equations considered, $E=T, R_{q} \subset J_{q}(T)$ is a transitive involutive system of infinitesimal Lie equations of order $q$ and the corresponding operator $\mathcal{D}$ is a Lie operator. As an exercise, we invite the reader to draw this diagram in the affine and projective 1-dimensional cases.

EXAMPLE 2.3: If we restrict our study to the group of isometries of the euclidean metric $\omega$ in dimension $n \geq 2$, exhibiting the Janet and the Spencer sequences is not easy at all, even when $n=2$, because the corresponding Killing operator

$$
\mathcal{D} \xi=\mathcal{L}(\xi) \omega=\Omega \in S_{2} T^{*},
$$

involving the Lie derivative $\mathcal{L}$ and providing twice the with $C_{r} \subset C_{r}(E)$, the linear Spencer sequence is induced by the linear hybrid sequence:

$$
0 \rightarrow E \stackrel{j_{q}}{\rightarrow} C_{0}(E) \stackrel{D_{1}}{\rightarrow} C_{1}(E) \stackrel{D_{2}}{\rightarrow} C_{2}(E) \stackrel{D_{3}}{\rightarrow} \cdots \stackrel{D_{n}}{\rightarrow} C_{n}(E) \rightarrow 0
$$

which is at the same time the Janet sequence for $j_{q}$ and the Spencer sequence for

$$
J_{q+1}(E) \subset J_{1}\left(J_{q}(E)\right)
$$

$[8,14]$. Such a sequence projects onto the Janet sequence and we have the following commutative diagram with exact columns:

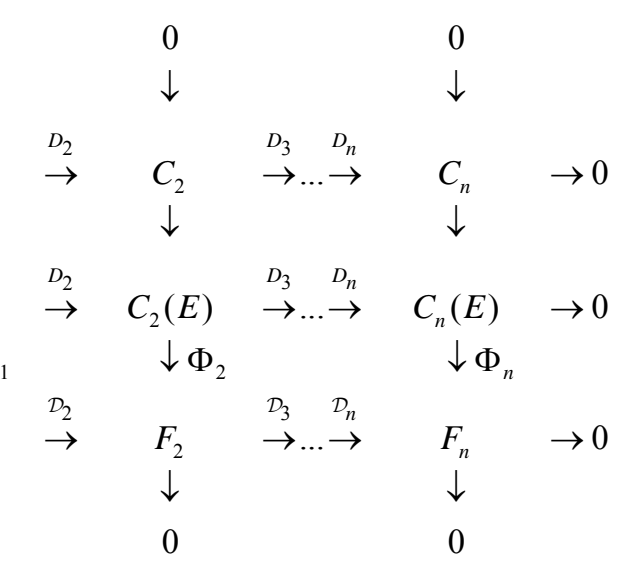

so-called infinitesimal deformation tensor $\varepsilon$ of continuum mechanics, is not involutive. In order to overcome this problem, one must differentiate once by considering also the Christoffel symbols $\gamma$ and add the operator

$$
\mathcal{L}(\xi) \gamma=\Gamma \in S_{2} T^{*} \otimes T .
$$

Now, one can prove that the Spencer sequence for Lie groups of transformations is locally isomorphic to the tensor product of the Poincaré sequence by the Lie algebra of the underlying Lie group [7,8]. Hence, if two Lie groups $G \subset \hat{G}$ act on $X$, it follows from the definition of the Janet and Spencer bundles that the Spencer sequence for $G$ is embedded into the Spencer sequence for $\hat{G}$ while the Janet sequence for $G$ projects onto the Janet sequence for $\hat{G}$ but the common differences are isomorphic to $\wedge^{r} T^{*} \otimes(\hat{\mathcal{G}} / \mathcal{G})$. This rather philosophical comment, namely to replace the Janet sequence by the Spencer sequence, must be considered as the crucial key for understanding the work of the brothers $\mathrm{E}$. and F. Cosserat in 1909 [7,17-19] or H. Weyl in 1918 [7,16], the best picture being that of Janet and Spencer playing at see-saw. Indeed, when $n=2$, one has 3 parameters (2 translations +1 rotation) and the following commutative diagram which only depends on the left commutative square: 


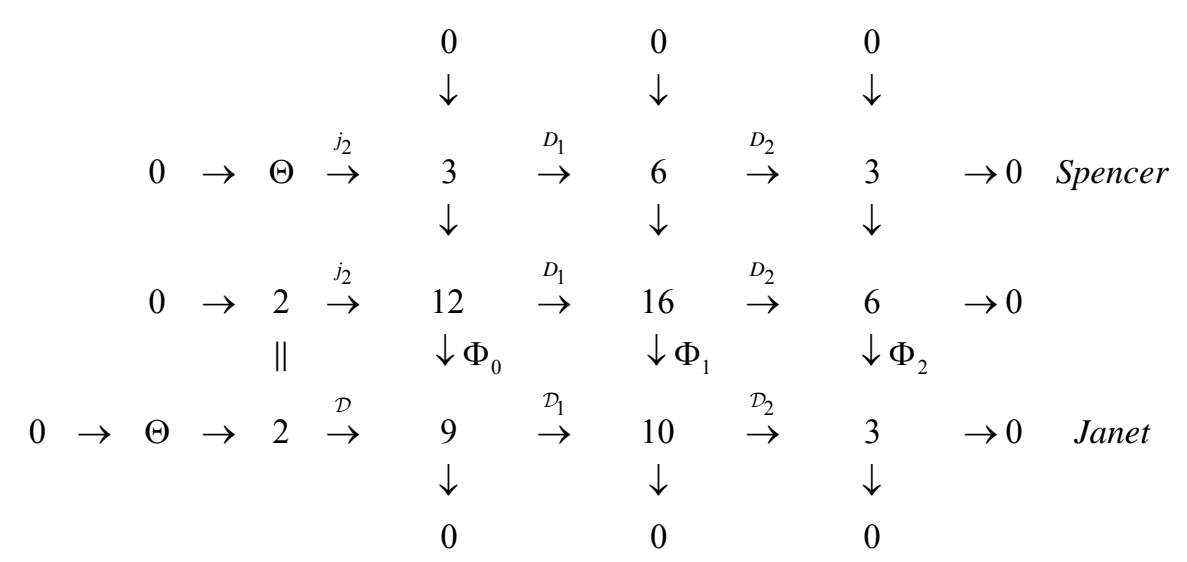

In this diagram, there is no way to compare $\mathcal{D}_{1}$ (curvature alone as in Vessiot) with $D_{2}$ (curvature + torsion as in Cartan).

For proving that the adjoint of $D_{1}$ provides the Cosserat equations which can be parametrized by the adjoint of $D_{2}$, we may lower the upper indices by means of the constant euclidean metric and look for the factors of $\xi_{1}, \xi_{2}$ and $\xi_{1,2}=-\xi_{2,1}$ in the integration by parts of the sum:

$$
\begin{aligned}
& \sigma^{11}\left(\partial_{1} \xi_{1}-\xi_{1,1}\right)+\sigma^{12}\left(\partial_{2} \xi_{1}-\xi_{1,2}\right)+\sigma^{21}\left(\partial_{1} \xi_{2}-\xi_{2,1}\right) \\
& +\sigma^{22}\left(\partial_{2} \xi_{2}-\xi_{2,2}\right)+\mu^{r}\left(\partial_{r} \xi_{1,2}-\xi_{1,2 r}\right)
\end{aligned}
$$

in order to obtain:

$$
\begin{aligned}
& \partial_{1} \sigma^{11}+\partial_{2} \sigma^{12}=f^{1}, \partial_{1} \sigma^{21}+\partial_{2} \sigma^{22}=f^{2}, \\
& \partial_{1} \mu^{1}+\partial_{2} \mu^{2}+\sigma^{12}-\sigma^{21}=m
\end{aligned}
$$

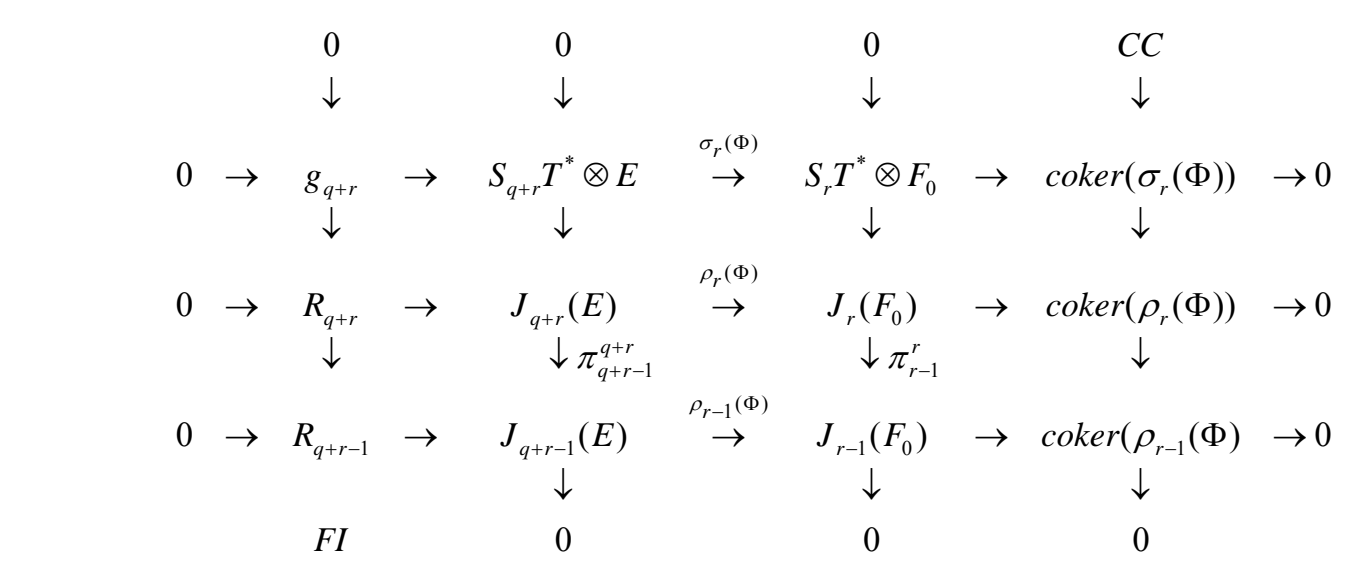

Finally, we get the nontrivial first order parametrization

$$
\begin{aligned}
\sigma^{11} & =\partial_{2} \phi^{1}, \sigma^{12}=-\partial_{1} \phi^{1}, \\
\sigma^{21} & =-\partial_{2} \phi^{2}, \sigma^{22}=\partial_{1} \phi^{2}, \\
\mu^{1} & =\partial_{2} \phi^{3}+\phi^{1}, \mu^{2}=-\partial_{1} \phi^{3}-\phi^{2}
\end{aligned}
$$

by means of the three arbitrary functions $\phi^{1}, \phi^{2}, \phi^{3}$, in a coherent way with the Airy second order parametrization obtained if we set

$$
\phi^{1}=\partial_{2} \phi, \phi^{2}=\partial_{1} \phi, \phi^{3}=-\phi
$$

when $\mu^{1}=0, \mu^{2}=0$ as we shall see in the third part.

The link between the FI of $R_{q}$ and the CC of $\mathcal{D}$ is expressed by the following diagram that may be used inductively:

The "snake theorem" $[8,20]$ then provides the long exact connecting sequence:

$$
0 \rightarrow g_{q+r} \rightarrow R_{q+r} \rightarrow R_{q+r-1} \rightarrow \operatorname{coker}\left(\sigma_{r}(\Phi)\right) \rightarrow \operatorname{coker}\left(\rho_{r}(\Phi)\right) \rightarrow \operatorname{coker}\left(\rho_{r-1}(\Phi)\right) \rightarrow 0 \text {. }
$$

If we apply such a diagram to first order Lie equations with no zero or first order CC, we have $q=1, E=T$ and we may apply the Spencer $\delta$-map to the top row obtained with $r=2$ in order to get the commutative diagram: 


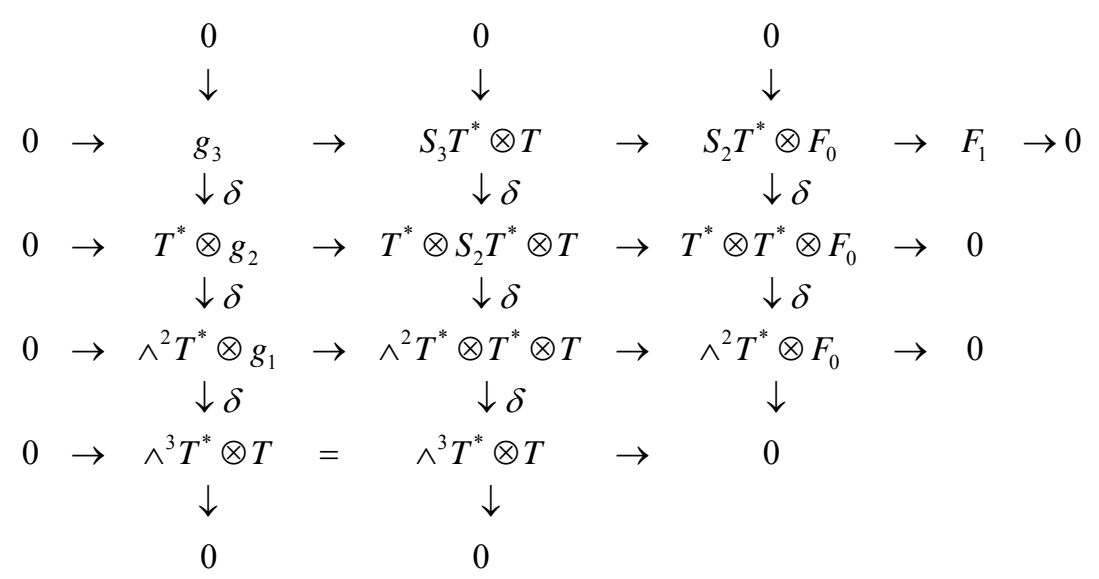

with exact rows and exact columns but the first that may not be exact at $\wedge^{2} T^{*} \otimes g_{1}$. We shall denote by $B^{2}\left(g_{1}\right)$ the coboundary as the image of the central $\delta$, by $Z^{2}\left(g_{1}\right)$ the cocycle as the kernel of the lower $\delta$ and by $H^{2}\left(g_{1}\right)$ the Spencer $\delta$-cohomology at $\wedge^{2} T^{*} \otimes g_{1}$ as the quotient.

In the classical Killing system, $g_{1} \subset T^{*} \otimes T$ is defined by

$$
\begin{aligned}
& \omega_{r j}(x) \xi_{i}^{r}+\omega_{i r}(x) \xi_{j}^{r}=0 \\
& \Rightarrow \xi_{r}^{r}=0, g_{2}=0, g_{3}=0
\end{aligned}
$$

Applying the previous diagram, we discover that the Riemann tensor is a section of the bundle

$$
\text { Riemann }=F_{1}=H^{2}\left(g_{1}\right)=Z^{2}\left(g_{1}\right)
$$

with

$$
\begin{aligned}
& \operatorname{dim}(\text { Riemann }) \\
= & \left(n^{2}(n+1)^{2} / 4\right)-\left(n^{2}(n+1)(n+2) / 6\right) \\
= & \left(n^{2}(n-1)^{2} / 4\right)-\left(n^{2}(n-1)(n-2) / 6\right) \\
= & n^{2}\left(n^{2}-1\right) / 12
\end{aligned}
$$

by using the top row or the left column. Though we discover the two properties of the Riemann tensor through the chase involved, we have no indices and cannot therefore exhibit the Ricci tensor of GR by means of the usual contraction or trace.

Let us proceed the same way with the conformal Killing system

$$
\begin{aligned}
\hat{\Omega}_{i j} & \equiv(\mathcal{L}(\xi) \hat{\omega})_{i j} \\
& \equiv \hat{\omega}_{r j} \partial_{i} \xi^{r}+\hat{\omega}_{i r} \partial_{j} \xi^{r}-\frac{2}{n} \omega_{i j} \partial_{r} \xi^{r}+\xi^{r} \partial_{r} \hat{\omega}_{i j}=0
\end{aligned}
$$

obtained by introducing

$$
\hat{\omega}_{i j}=\omega_{i j} / \mid \operatorname{det}(\omega)^{\frac{1}{n}}
$$

or, equivalently, by eliminating $A(x)$ in

$$
\mathcal{L}(\xi) \omega=A(x) \omega .
$$

Now $\hat{g}_{1}$ is defined by

$$
\omega_{r j} \xi_{i}^{r}+\omega_{i r} \xi_{j}^{r}-\frac{2}{n} \omega_{i j} \xi_{r}^{r}=0
$$

but we have $\hat{g}_{3}=0, \forall n \geq 3$ with $H^{2}\left(\hat{g}_{2}\right)=0, \forall n \geq 4$ and the Weyl tensor is a section of the bundle

$$
\text { Weyl }=\hat{F}_{1}=H^{2}\left(\hat{g}_{1}\right)=Z^{2}\left(\hat{g}_{1}\right) / \delta\left(T^{*} \otimes \hat{g}_{2}\right)
$$

with

$$
\operatorname{dim}(\text { Weyl })=n(n+1)(n+2)(n-3) / 12 .
$$

Similarly, we have no indices and cannot therefore exhibit the Ricci tensor. However, when $n=4$, among the components of the Spencer operator we have

$$
\partial_{i} \xi_{r j}^{r}-\xi_{r i j}^{r}=\partial_{i} \xi_{r j}^{r}
$$

and thus

$$
\partial_{i} \xi_{r j}^{r}-\partial_{j} \xi_{r i}^{r}=F_{i j} .
$$

Such a result allows to recover the electromagnetic field in the image of the Spencer operator $D_{1}$ and Maxwell equations by duality along the way proposed by Weyl in [16] but the use of the Spencer operator provides the only possibility to exhibit a link with Cosserat equations.

Comparing the classical and conformal Killing systems by using the inclusions

$$
R_{1} \subset \hat{R}_{1} \Rightarrow g_{1} \subset \hat{g}_{1},
$$

we finally obtain the following commutative and exact diagram where a diagonal chase allows to identify Ricci with

$$
S_{2} T^{*} \subset T^{*} \otimes T^{*} \simeq T^{*} \otimes \hat{g}_{2}
$$

and to split the right column $[7,12,20]$ : 


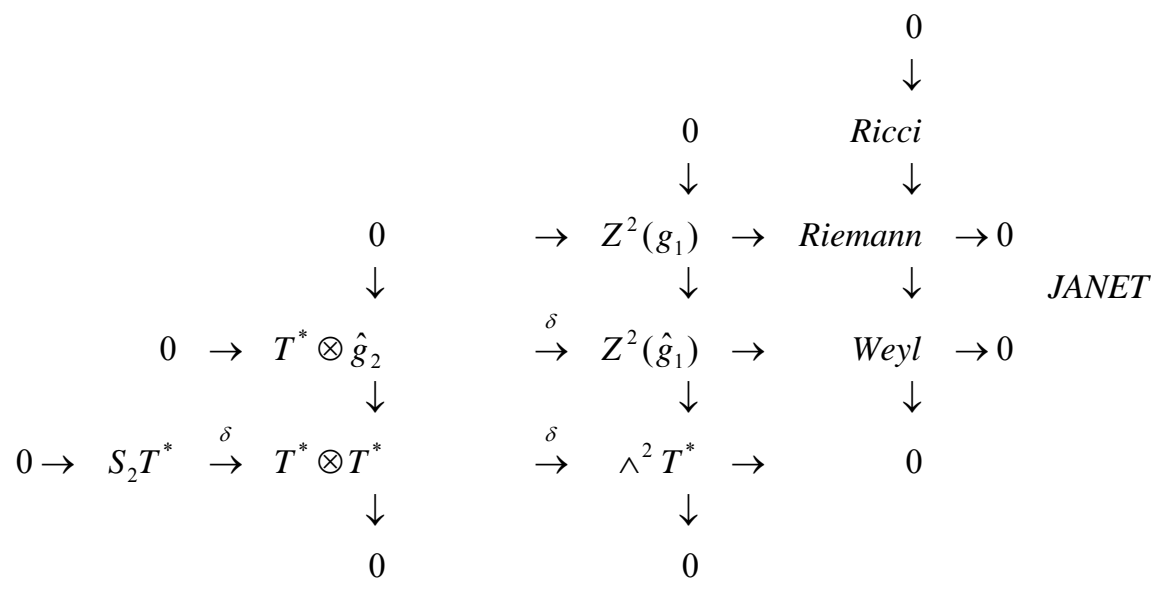

SPENCER

SECOND FUNDAMENTAL RESULT: The Ricci tensor only depends on the "difference" existing between the clasical Killing system and the conformal Killing system, namely the $n$ second order jets (elations once more). The Ricci tensor, thus obtained without contracting the indices as usual, may be embedded in the image of the Spencer operator made by 1 -forms with value in 1 -forms that we have already exhibited for describing EM. It follows that the foundations of both EM and GR are not coherent with jet theory and must therefore be revisited within this new framework.

\section{Third Part: Algebraic Analysis}

EXAMPLE 3.1: Let a rigid bar be able to slide along an horizontal axis with reference position $x$ and attach two pendula, one at each end, with lengths $l_{1}$ and $l_{2}$, having small angles $\theta_{1}$ and $\theta_{2}$ with respect to the vertical. If we project Newton law with gravity $g$ on the perpendicular to each pendulum in order to eliminate the tension of the threads and denote the time derivative with a dot, we get the two equations:

$$
\ddot{x}+l_{1} \ddot{\theta}_{1}+g \theta_{1}=0, \ddot{x}+l_{2} \ddot{\theta}_{2}+g \theta_{2}=0
$$

As an experimental fact, starting from an arbitrary movement of the pendula, we can stop them by moving the bar if and only if $l_{1} \neq l_{2}$ and we say that the system is controllable.

More generally, we can bring the OD equations describing the behaviour of a mechanical or electrical system to the Kalman form $\dot{y}=A y+b u$ with input $u=\left(u^{1}, \cdots, u^{p}\right)$ and output $y=\left(y^{1}, \cdots, y^{m}\right)$. We say that the system is controllable if, for any given

$$
y(0), y(T), T<\infty,
$$

one can find $u(t)$ such that a coherent trajectory $y(t)$ may be found. In 1963 [21], R. E. Kalman discovered that the system is controllable if and only if

$$
r k\left(B, A B, \cdots, A^{m-1} B\right)=m .
$$

Surprisingly, such a functional definition admits a formal test which is only valid for Kalman type systems with constant coefficients and is thus far from being intrinsic. In the PD case, the Spencer form will replace the Kalman form.

\section{EXAMPLE 3.2:}

$$
\begin{aligned}
& \dot{y}-\dot{u}=0 \Rightarrow y(t)-u(t)=c=c s t \\
& \Rightarrow u(T)-u(0)=y(T)-y(0)
\end{aligned}
$$

can always be achieved and the system is thus controllable in the sense of the definition but $z=y-u \Rightarrow \dot{z}=0$ is not controllable in the sense of the test.

EXAMPLE 3.3:

$$
\dot{y}^{1}-a(t) y^{2}-\dot{y}^{3}=0, y^{1}-\dot{y}^{2}+\dot{y}^{3}=0 .
$$

Any way to bring this system to Kalman form provides the controllability condition $a(a-1) \neq 0$ if $a=c s t$ but nothing can be said if $a=a(t)$. Also, getting $y^{1}$ from the second equation and substituting in the first, we get the second order OD equation

$$
\ddot{y}^{2}-\ddot{y}^{3}-\dot{y}^{3}-a(t) y^{2}=0
$$

for which nothing can be said at first sight.

PROBLEM 1: Is a SYSTEM of OD or PD equations "controllable" (answer must be YES or NO) and how can we define controllability?

Now, if a differential operator $\stackrel{\mathcal{D}}{\rightarrow} \eta$ is given, a direct problem is to find (generating) compatibility conditions (CC) as an operator $\eta \stackrel{\mathcal{D}_{1}}{\rightarrow} \zeta$ such that

$$
\mathcal{D} \xi=\eta \Rightarrow \mathcal{D}_{1} \eta=0
$$

Conversely, the inverse problem will be to find $\stackrel{\mathcal{D}_{-1}}{\rightarrow} \xi$ 
such that $\mathcal{D}$ generates the $\mathrm{CC}$ of $\mathcal{D}_{-1}$ and we shall say that $\mathcal{D}$ is parametrized by $\mathcal{D}_{-1}$. Of course, solving the direct problem (Janet, Spencer) is necessary for solving the inverse problem.

EXAMPLE 3.4: When $n=2$, the Cauchy equations for the stress in continuum mechanics are

$$
\partial_{1} \sigma^{11}+\partial_{2} \sigma^{21}=0, \partial_{1} \sigma^{12}+\partial_{2} \sigma^{22}=0
$$

with $\sigma^{12}=\sigma^{21}$.

Their parametrization

$$
\sigma^{11}=\partial_{22} \phi, \sigma^{12}=\sigma^{21}=-\partial_{12} \phi, \sigma^{22}=\partial_{11} \phi
$$

has been discovered by Airy in 1862 and $\phi$ is called the Airy function. When $n=3$, Maxwell and Morera discovered a similar parametrization with 3 potentials (exercise).

EXAMPLE 3.5: When $n=4$, the Maxwell equations $d F=0$ where $F \in \wedge^{2} T^{*}$ is the EM field are parametrized by $d A=F$ where $A \in T^{*}$ is the 4-potential. The second set of Maxwell equations can also be parametrized by the so-called pseudopotential which is a pseudovector density (exercise).

EXAMPLE 3.5: If $n=4, \omega$ is the Minkowski metric and $\phi=G M / r$ is the gravitational potential, then $\phi / c^{2} \ll 1$ and a perturbation $\Omega$ of $\omega$ may satisfy in vacuum the 10 second order Einstein equations for the $10 \Omega$ :

$$
\begin{aligned}
& \omega^{r s}\left(d_{i j} \Omega_{r s}+d_{r s} \Omega_{i j}-d_{r i} \Omega_{s j}-d_{s j} \Omega_{r i}\right) \\
& -\omega_{i j}\left(\omega^{r s} \omega^{u v} d_{r s} \Omega_{u v}-\omega^{r u} \omega^{s v} d_{r s} \Omega_{u v}\right)=0
\end{aligned}
$$

The parametrizing challenge has been proposed in 1970 by J. Wheeler for $1000 \$$ and solved negatively in 1995 by the author who only received $1 \$$.

PROBLEM 2: Is an OPERATOR parametrizable (answer must be YES or NO) and how can we find a parametrization?

Let $A$ be a unitary ring, that is

$$
1, a, b \in A \Rightarrow a+b, a b \in A, 1 a=a
$$

and even an integral domain, that is

$$
a b=0 \Rightarrow a=0 \text { or } b=0 \text {. }
$$

We say that $M={ }_{A} M$ is a left module over $A$ if

$$
x, y \in M \Rightarrow a x, x+y \in M, \forall a \in A
$$

and we denote by $\operatorname{hom}_{A}(M, N)$ the set of morphisms $f: M \rightarrow N$ such that $f(a x)=a f(x)$.

DEFINITION 3.6: We define the torsion submodule

$$
t(M)=\{x \in M \mid \exists 0 \neq a \in A, a x=0\} \subseteq M .
$$

There is a sequence

$$
0 \rightarrow t(M) \rightarrow M \stackrel{\varepsilon}{\rightarrow} \operatorname{hom}_{A}\left(\operatorname{hom}_{A}(M, A), A\right)
$$

where the morphism $\varepsilon$ is defined by

$$
\varepsilon(x)(f)=f(x), \forall x \in M, \forall f \in \operatorname{hom}_{A}(M, A)
$$

because we have at once

$$
\text { af }(x)=f(a x)=f(0)=0 \Rightarrow t(M) \subseteq \operatorname{ker}(\varepsilon) .
$$

PROBLEM 3: Is a MODULE $M$ torsion-free, that is $t(M)=0$ (answer must be YES or NO) and how can we test such a property?

In the remaining of this paper we shall prove that the three problems are indeed identical and that only the solution of the third will provide the solution of the two others [1,22-24].

Let $\mathbb{Q} \subset K$ be a differential field, that is a field

$$
(a \in K \Rightarrow 1 / a \in K)
$$

with $n$ commuting derivations $\left\{\partial_{1}, \cdots, \partial_{n}\right\}$ with

$$
\partial_{i} \partial_{j}=\partial_{j} \partial_{i}=\partial_{i j}, \forall i, j=1, \cdots, n
$$

such that

$$
\partial_{i}(a+b)=\partial_{i} a+\partial_{i} b
$$

and

$$
\partial_{i}(a b)=\left(\partial_{i} a\right) b+a \partial_{i} b, \forall a, b \in K .
$$

Using an implicit summation on multiindices, we may introduce the (noncommutative) ring of differential operators

$$
D=K\left[d_{1}, \cdots, d_{n}\right]=K[d]
$$

with elements $P=a^{\mu} d_{\mu}$ such that $|\mu|<\infty$ and $d_{i} a=$ $a d_{i}+\partial_{i} a$. Now, if we introduce differential indeterminates $y=\left(y^{1}, \cdots, y^{m}\right)$, we may extend $d_{i} y_{\mu}^{k}=y_{\mu+1_{i}}^{k}$ to

$$
\Phi^{\tau} \equiv a_{k}^{\tau \mu} y_{\mu}^{k} \stackrel{d_{i}}{\rightarrow} d_{i} \Phi^{\tau} \equiv a_{k}^{\tau \mu} y_{\mu+1_{i}}^{k}+\partial_{i} a_{k}^{\tau \mu} y_{\mu}^{k}
$$

for $\tau=1, \cdots, p$. Therefore, setting

$$
D y^{1}+\cdots+d y^{m}=D y \simeq D^{m},
$$

we obtain by residue the differential module or $D$ module $M=D y / D \Phi$. Introducing the two free differential modules $F_{0} \simeq D^{m}, F_{1} \simeq D^{p}$, we obtain equivalently the free presentation $F_{1} \stackrel{\mathcal{D}}{\rightarrow} F_{0} \rightarrow M \rightarrow 0$. More generally, introducing the successive $\mathrm{CC}$ as in the preceding section, we may finally obtain the free resolution of $M$, namely the exact sequence

$$
\stackrel{\mathcal{D}_{2}}{\rightarrow} F_{2} \stackrel{\mathcal{D}_{1}}{\rightarrow} F_{1} \stackrel{\mathcal{D}}{\rightarrow} F_{0} \rightarrow M \rightarrow 0 .
$$

The "trick" is to let $\mathcal{D}$ act on the left on column vectors in the operator case and on the right on row vectors in the module case. Homological algebra has been created for finding intrinsic properties of modules not depending on their presentation or even on their resolution. 
EXAMPLE 3.7: In order to understand that different presentations may nevertheless provide isomorphic modules, let us consider the linear inhomogeneous system $P y \equiv d_{22} y=u, Q \equiv d_{12} y-y=v$ with $K=\mathbb{Q}$. Differentiating twice, we get $y=d_{11} u-d_{12} v-v$ and the two fourth order $\mathrm{CC}$ :

$$
\begin{aligned}
& A \equiv d_{1122} u-d_{1222} v-d_{22} v-u=0, \\
& B \equiv d_{1112} u-d_{1122} v-d_{11} u=0 \Rightarrow E \\
& \equiv d_{12} B-d_{11} A+B=0 .
\end{aligned}
$$

However, as $P Q=Q P$, we also get the $C C$ $C \equiv d_{12} u-d_{22} v-u=0$ and the two resolutions:

$$
\begin{aligned}
& 0 \rightarrow D \rightarrow D^{2} \rightarrow D^{2} \rightarrow M \rightarrow 0, \\
& 0 \rightarrow D \rightarrow D^{2} \rightarrow M \rightarrow 0
\end{aligned}
$$

where we can identify the two differential modules involved on the right with $D$ because:

$$
\begin{aligned}
& A \equiv d_{12} C+C, \\
& B \equiv d_{11} C \Leftrightarrow C \equiv d_{22} B-d_{12} A+B=0 .
\end{aligned}
$$

We now exhibit another approach by defining the formal adjoint of an operartor $P$ and an operator matrix $\mathcal{D}$ :

DEFINITION 3.8:

$$
\begin{aligned}
& P=a^{\mu} d_{\mu} \in D \stackrel{a d}{\leftrightarrow} a d(P)=(-1)^{|\mu|} d_{\mu} a^{\mu} \in D \\
& \langle\lambda, \mathcal{D} \xi\rangle=\langle\operatorname{ad}(\mathcal{D}) \lambda, \xi\rangle+\operatorname{div}(\cdots)
\end{aligned}
$$

from integration by part, where $\lambda$ is a row vector of test functions and \langle\rangle the usual contraction.

PROPOSITION 3.9: If we have an operator $E \stackrel{\mathcal{D}}{\rightarrow} F$, we obtain by duality an operator

$$
\wedge^{n} T^{*} \otimes E^{*} \stackrel{a d(\mathcal{D})}{\leftarrow} \wedge^{n} T^{*} \otimes F^{*}
$$

where $E^{*}$ is obtained from $E$ by inverting the transition matrix and EM provides a fine example of such a procedure [10].

Now, with operational notations, let us consider the two differential sequences:

$$
\begin{aligned}
& \stackrel{\mathcal{D}}{\rightarrow} \eta \stackrel{\mathcal{D}_{1}}{\rightarrow} \zeta \\
& \stackrel{\operatorname{ad}(\mathcal{D})}{\leftarrow} \mu \stackrel{\operatorname{ad}\left(\mathcal{D}_{1}\right)}{\leftarrow} \lambda
\end{aligned}
$$

where $\mathcal{D}_{1}$ generates all the $\mathrm{CC}$ of $\mathcal{D}$. Then

$$
\mathcal{D}_{1} \circ \mathcal{D} \equiv 0 \Leftrightarrow \operatorname{ad}(\mathcal{D}) \circ \operatorname{ad}\left(\mathcal{D}_{1}\right) \equiv 0
$$

but $\operatorname{ad}(\mathcal{D})$ may not generate all the CC of $\operatorname{ad}\left(\mathcal{D}_{1}\right)$.

EXAMPLE 3.10: With $\partial_{22} \xi=\eta^{2}, \partial_{12} \xi=\eta^{1}$ for $\mathcal{D}$, we get $\partial_{1} \eta^{2}-\partial_{2} \eta^{1}=\zeta$ for $\mathcal{D}_{1}$. Then $\operatorname{ad}\left(\mathcal{D}_{1}\right)$ is defined by $\mu^{2}=-\partial_{1} \lambda, \mu^{1}=\partial_{2} \lambda$ while $\operatorname{ad}(\mathcal{D})$ is defined by $v=\partial_{12} \mu^{1}+\partial_{22} \mu^{2}$ but the $\mathrm{CC}$ of $\operatorname{ad}\left(\mathcal{D}_{1}\right)$ are generated by $v^{\prime}=\partial_{1} \mu^{1}+\partial_{2} \mu^{2}$. Passing to the module framework, we obtain the sequences:

$$
\begin{aligned}
& D \stackrel{\mathcal{D}_{1}}{\rightarrow} D^{2} \stackrel{\mathcal{D}}{\rightarrow} D \quad D \quad M \rightarrow 0 \\
& D \stackrel{\operatorname{ad}\left(\mathcal{D}_{1}\right)}{\leftarrow} \quad D^{2} \stackrel{\operatorname{ad}(\mathcal{D})}{\leftarrow} \quad D
\end{aligned}
$$

THEOREM 3.11: The cohomology $\operatorname{ext}^{1}(M)$ at $D^{2}$ of the lower sequence does not depend on the resolution of $M$ and is a torsion module called the first extension module of $M$.

Exactly like we defined the differential module $M$ from $\mathcal{D}$, let us define the differential module $N$ from $\operatorname{ad}(\mathcal{D})$. The proof of the next theorem is quite tricky and out of the scope of this paper [1,22-24]:

MAIN THEOREM 3.12: $\operatorname{ext}^{1}(N)=t(M)=\operatorname{ker}(\varepsilon)$.

FORMAL TEST 3.13: The double duality test needed in order to check whether $t(M)=0$ or not and to find out a parametrization if $t(M)=0$ has 5 steps which are drawn in the following diagram where $\operatorname{ad}\left(\mathcal{D}_{-1}\right)$ generates the $\mathrm{CC}$ of $\operatorname{ad}(\mathcal{D})$ and $\mathcal{D}^{\prime}$ generates the $\mathrm{CC}$ of $\mathcal{D}_{-1}$ :

$$
\begin{aligned}
& \eta^{\prime} \quad 5 \\
& \mathcal{D}^{\prime} \\
& 4 \quad \theta \stackrel{\mathcal{D}_{-1}}{\rightarrow} \xi \stackrel{\mathcal{D}}{\rightarrow} \quad \eta \quad 1 \\
& 3 \quad v \stackrel{\operatorname{ad}\left(\mathcal{D}_{-1}\right)}{\leftarrow} \mu \stackrel{\operatorname{ad}(\mathcal{D})}{\leftarrow} \quad \lambda \quad 2
\end{aligned}
$$

THEOREM 3.14: $\mathcal{D}$ parametrized by

$$
\mathcal{D}_{-1} \Leftrightarrow \mathcal{D}=\mathcal{D}^{\prime} \Leftrightarrow t(M)=0 \Leftrightarrow \operatorname{ext}^{1}(N)=0 .
$$

COROLLARY 3.15: If $n=1$ and $\mathcal{D}$ is surjective, then $t(M)=0$ if and only if $\operatorname{ad}(\mathcal{D})$ is injective $[24,25]$.

EXAMPLE 3.16: (Kalman test revisited) If we multiply the Kalman system $-\dot{y}+A y+B u=0$ on the left by a test row vector $\lambda$, we obtain:

$$
\operatorname{ad}(\mathcal{D})\left\{\begin{array}{rrr}
y & \rightarrow & \dot{\lambda}+\lambda A=0 \\
u & \rightarrow & \lambda B=0
\end{array}\right.
$$

Differentiating the zero order equations and using the first order ones, we get $\lambda A B=0$ and so on. Using the Cayley-Hamilton theorem, we stop at $\lambda A^{m-1} B=0$ and find back exactly the Kalman test but in a completely different intrinsic framework.

EXAMPLE 3.17: (Double pendulum revisited) Using two test functions $\lambda^{1}$ and $\lambda^{2}$, we get:

$$
\operatorname{ad}(\mathcal{D})\left\{\begin{array}{llr}
x & \rightarrow & \ddot{\lambda}^{1}+\ddot{\lambda}^{2}=0 \\
\theta_{1} & \rightarrow & l_{1} \ddot{\lambda}^{1}+g \lambda^{1}=0 \\
\theta_{2} & \rightarrow & l_{2} \ddot{\lambda}^{2}+g \lambda^{2}=0
\end{array}\right.
$$


and obtain at once the zero order equation

$$
l_{1} \lambda^{2}+l_{2} \lambda^{1}=0
$$

Differentiating twice and substituting, we also get $\left(l_{1} / l_{2}\right) \lambda^{2}+\left(l_{2} / l_{1}\right) \lambda^{1}=0$ and $\operatorname{ad}(\mathcal{D})$ is injective if and only if $l_{2}-l_{1} \neq 0$.

EXAMPLE 3.18: (Airy parametrization revisited) When $n=2$, we may study the infinitesimal deformation $\varepsilon \in S_{2} T^{*}$ by means of the Killing operator $\mathcal{D} \xi \equiv \mathcal{L}(\xi) \omega=2 \varepsilon$ when $\omega$ is the euclidean metric. Then $\operatorname{ad}(\mathcal{D})$ provides (up to sign and factor 2 ) the Cauchy equations $\partial_{r} \sigma^{r j}+\gamma_{r s}^{j} \sigma^{r s}=0$ for the stress tensor density $[12,16,26]$. The following diagram describes the Poincaré scheme:

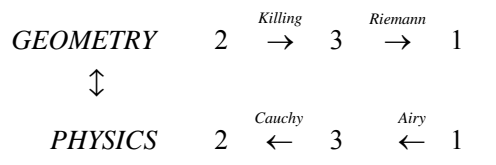

Accordingly, the second order Airy parametrization is nothing else than the adjoint of the only Riemann $\mathrm{CC}$ involved, namely $\partial_{11} \varepsilon_{22}+\partial_{22} \varepsilon_{11}-2 \partial_{12} \varepsilon_{12}=0$ which is the linearization of the Riemann tensor of Example 1.9.

EXAMPLE 3.19: (Einstein equations revisited) Contrary to the Ricci operator, the Einstein operator is selfadjoint because it comes from a variational procedure, the sixth terms being exchanged between themselves under ad . For example, we have:

$$
\lambda^{i j}\left(\omega^{r s} d_{i j} \Omega_{r s}\right) \stackrel{a d}{\leftrightarrow}\left(\omega^{r s} d_{i j} \lambda^{i j}\right) \Omega_{r s}=\left(\omega_{i j} d_{r s} \lambda^{r s}\right) \Omega^{i j}
$$

and the adjoint of the first operator is the sixth. Accordingly, one has the following diagram where $\mathcal{D} \neq \mathcal{D}^{\prime}$ :

$$
\begin{aligned}
& \text { Riemann } \\
& 20 \\
& 4 \stackrel{\text { Killing }}{\rightarrow} \quad 10 \stackrel{\nearrow}{\rightarrow} \quad 10 \\
& 4 \stackrel{\text { Cauchy }}{\leftarrow} 10 \stackrel{\text { Einstein }}{\leftarrow} 10
\end{aligned}
$$

THIRD FUNDAMENTAL RESULT: Comparing this diagram to the previous one proves that Einstein equations are not coherent with Janet and Spencer sequences as conformal geometry has not been introduced in this last part.

EXERCISE 3.20: Prove that

$$
\ddot{y}^{2}-\ddot{y}^{3}-\dot{y}^{3}-a(t) y^{2}=0
$$

is controllable if and only if $\dot{a}+a^{2}-a \neq 0$ (Riccati) and find a parametrization.

EXERCISE 3.21: Prove that the infinitesimal contact transformations of Example 1.10 admit the injective parametrization

$$
\begin{aligned}
& -x^{3} \partial_{3} \theta+\theta=\xi^{1}, \quad-\partial_{3} \theta=\xi^{2}, \\
& \partial_{2} \theta-x^{3} \partial_{1} \theta=\xi^{3} \Rightarrow \xi^{1}-x^{3} \xi^{2}=\theta .
\end{aligned}
$$

\section{Conclusion}

The mathematical foundations of General Relativity leading to Einstein equations are always presented in textbooks or papers without any reference to conformal geometry. However, comparing the classical Killing equations to the conformal Killing equations while constructing corresponding differential sequences, the Ricci tensor appears as the kernel of the canonical projection of the Riemann tensor onto the Weyl tensor. After obtaining such a result in a purely intrinsic way, that is without using indices, we have been able to introduce "diagram chasing" in order to relate for the first time electromagnetism and gravitation to the Spencer $\delta$-cohomology of the classical and conformal Killing symbols. Accordingly, the mathematical foundations of general relativity are not coherent with jet theory and must therefore be revisited within this new framework along the lines we have sketched. Finally, the fact that Einstein equations cannot be parametrized, contrary to most other equations of physics or engineering, also brings a deep structural question on these equations that will have to be solved in the future by means of algebraic analysis.

\section{REFERENCES}

[1] J.-F. Pommaret, "Partial Differential Control Theory," Kluwer, Dordrecht, 2001. doi:10.1007/978-94-010-0854-9

[2] E. Cartan, Annales Scientifiques de l'École Normale Supérieure, Vol. 21, 1904, pp. 153-206.

[3] E. Cartan, Annales Scientifiques de l'École Normale Supérieure, Vol. 40, 1923, pp. 325-412.

[4] H. Goldschmidt, Journal of Differential Geometry, Vol. 6, 1972, pp. 357-373,

[5] A. Kumpera and D. C. Spencer, "Lie Equations," Annals of Mathematics Studies 73, Princeton University Press, Princeton, 1972.

[6] J.-F. Pommaret, "Differential Galois Theory," Gordon and Breach, New York, 1983.

[7] J.-F. Pommaret, "Spencer Operator and Applications: From Continuum Mechanics to Mathematical Physics," In: Y. Gan, Ed., Continuum Mechanics-Progress in Fundamentals and Engineering Applications, InTech, 2012. http://www.intechopen.com/books/continuum-mechanicspro-

gress-in-fundamentals-and-engineering-applications/spen cer-operator-and-applications-from-continuum-mechanics -to-mathematical-physics

[8] J.-F. Pommaret, "Partial Differential Equations and Group Theory," Kluwer, 1994. doi:10.1007/978-94-017-2539-2 
[9] E. Vessiot, Annales scientifiques de l'École Normale Supérieure, Vol. 20, 1903, pp. 411-451.

[10] V. Ougarov, "Théorie de la Relativité Restreinte," MIR, Moscow, 1969.

[11] L. P. Eisenhart, "Riemannian Geometry," Princeton University Press, Princeton, 1926.

[12] J.-F. Pommaret, "Lie Pseudogroups and Mechanics," Gordon and Breach, New York, 1988.

[13] A. Lorenz, "Jet Groupoids, Natural Bundles and the Vessiot Equivalence Method," Acta Applicandae Mathematicae, Vol. 1, 2008, pp. 205-213. doi:10.1007/s10440-008-9193-7

[14] D. C. Spencer, Bulletin of the American Mathematical Society, Vol. 75, 1965, pp. 1-114.

[15] M. Janet, Journal de Mathématiques Pures et Appliquées, Vol. 8, 1920, pp. 65-151.

[16] H. Weyl, "Space, Time, Matter," Berlin, 1918.

[17] E. Cosserat and F. Cosserat, "Théorie des Corps Déformables," Hermann, Paris, 1909.

[18] J.-F. Pommaret, Acta Mechanica, Vol. 149, 2001, pp. 23-39. doi:10.1007/BF01261661
[19] J.-F. Pommaret, Acta Mechanica, Vol. 215, 2010, pp. 4355. doi:10.1007/s00707-010-0292-y

[20] J. J. Rotman, “An Introduction to Homological Algebra," Academic Press, Cambridge, 1979.

[21] R. E. Kalman, Y. C. Yo and K. S. Narenda, Journal of Differential Equations, Vol. 1, 1963, pp. 189-213.

[22] M. Kashiwara, Mémoires de la Société Mathématique de France, Vol. 63, 1995, pp. 1-72.

[23] V. P. Palamodov, "Linear Differential Operators with Constant Coefficients," Grundlehren der Mathematischen Wissenschaften 168, Springer, 1970. doi:10.1007/978-3-642-46219-1

[24] J.-F. Pommaret, “Algebraic Analysis of Control Systems Defined by Partial Differential Equations," Advanced Topics in Control Systems Theory, Springer, Lecture Notes in Control and Information Sciences 311, 2005, pp. $155-223$

[25] E. Kunz, "Introduction to Commutative Algebra and Algebraic Geometry," Birkhäuser, 1985.

[26] W. Pauli, “Theory of Relativity,” Pergamon Press, London, 1958. 